Sel $f$-consi st ent l ong- $t i$ me si mil at $i$ on of chi rping and beating energetic particle rodes i n JT- 60U pl asmas

\begin{tabular}{|l|l|}
\hline $\begin{array}{l}j \text { our nal or } \\
\text { publ i cat } i \text { on } \mathrm{t} \text { i } \mathrm{tl} \text { e }\end{array}$ & Nucl ear Fusi on \\
\hline vol une & 57 \\
\hline number & 1 \\
\hline page $\mathrm{r}$ ange & 016036 \\
\hline year & 2016- 12 - 08 \\
\hline URL & ht t p: //hdl . handl e. net /10655/00012818 \\
\hline
\end{tabular}




\title{
Self-consistent long-time simulation of chirping and beating energetic particle modes in JT-60U plasmas
}

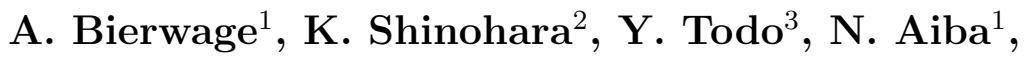 \\ M. Ishikawa ${ }^{2}$, G. Matsunaga ${ }^{2}$, M. Takechi ${ }^{2}$ and M. Yagi ${ }^{1}$ \\ 1 National Institutes for Quantum and Radiological Science and Technology, \\ Rokkasho Fusion Institute, Aomori 039-3212, Japan \\ 2 National Institutes for Quantum and Radiological Science and Technology, Naka \\ Fusion Institute, Ibaraki 311-0193, Japan \\ 3 National Institute for Fusion Science, Toki, Gifu 509-5292, Japan
}

\begin{abstract}
Recurring bursts of chirping Alfvén modes that were observed in JT-60U tokamak plasmas driven by negative-ion-based neutral beams (N-NB) are reproduced in first-principle simulations performed with an extended version of the hybrid code MEGA. This code simulates the interactions between gyrokinetic fast ions and magnetohydrodynamic (MHD) modes in the presence of a realistic fast ion source and collisions, so that it self-consistently captures dynamics across a wide range of time scales $(0.01-100 \mathrm{~ms})$. The simulation confirms that the experimentally observed phenomena known as "fast frequency sweeping (fast FS) modes" are caused by bursts of energetic particle modes (EPM) with dominant toroidal mode number $n=1$. On the long time scale $(1-10 \mathrm{~ms})$, the simulation reproduces the chirping range $(40-60 \mathrm{kHz})$, the burst duration (few $\mathrm{ms}$ ) and intervals $(5-10 \mathrm{~ms})$. On the short time scale (0.01-0.1 $\mathrm{ms}$ ), it reproduces pulsations and phase jumps, which we interpret as the result of beating between multiple resonant wave packets. Having reproduced at multiple levels of detail the dynamics of low-amplitude long-wavelength Alfvén modes driven by N-NB ions, the next goal is to reproduce and explain abrupt large-amplitude events (ALE) that were seen in the same experiments at longer time intervals $(10-100 \mathrm{~ms})$.
\end{abstract}

\section{Introduction}

In tokamak experiments with strong drive from fast ions, which are performed with the goal of exploring burning-plasma-relevant regimes, one often sees intermittent bursts of magnetohydrodynamic (MHD) wave activity as well as abrupt relaxation events. For instance, when powerful negative-ion-based neutral beams (N-NB) were injected into high-beta JT-60U tokamak plasmas, so-called "fast frequency sweeping modes" (fast FS modes) and "abrupt large-amplitude events" (ALE) were routinely observed $[1,2,3]$ and found to cause a significant amount of fast ion transport $[4,5]$. Motivated by these observations, the goals of the simulation study reported in the present paper and related future publications are as follows: 
(i) Reproduce the bursts of chirping modes and ALEs using numerical simulations and, at the same time, validate the simulation code and mathematical model against experimental measurements of MHD wave activity and fast ion transport.

(ii) Use the results of the validated first-principle simulations to study, interpret and explain the physical mechanisms that are responsible for the observations.

(iii) Pave the way for making reliable predictions for fast ion confinement in tokamak experiments and burning plasmas, including the construction of reduced models to be used for quick parameter surveys and the development of operational scenarios.

These are challenging tasks, because we have to assume that many of the relevant processes can act and interact on comparable or overlapping time scales; namely,

$$
\tau_{\text {wave }} \lesssim \tau_{\text {bounce }} \sim \tau_{\text {pulse }} \lesssim \tau_{\text {coll }} \sim \tau_{\text {src }}<\tau_{\text {equil }}
$$

The relation $\tau_{\text {wave }} \lesssim \tau_{\text {bounce }} \sim \tau_{\text {pulse }}$ means that the resonant instabilities and the associated fast ion transport may occur in short pulses, whose length $\tau_{\text {pulse }}$ is comparable to the bounce cycle $\tau_{\text {bounce }}$ of fast ions that are resonantly trapped inside MHD waves. Moreover, both $\tau_{\text {pulse }}$ and $\tau_{\text {bounce }}$ may last only a few $(\lesssim 10)$ wave oscillation periods $\tau_{\text {wave }}$. By allowing the three time scales $\tau_{\text {wave }} \lesssim \tau_{\text {bounce }} \sim \tau_{\text {pulse }}$ to overlap, one allows for short but intense MHD wave activity. Concerning the right half of Eq. (1), note that the primary role of collisions and sources is to fill and refill resonant regions in fast ion phase space $(\boldsymbol{x}, \boldsymbol{v})$. Collisions also contribute to the draining of resonances and decorrelation of wave-particle interactions. Consequently, their time scales $\tau_{\text {coll }}$ and $\tau_{\text {src }}$ vary as widely as the widths of resonances $(\delta \boldsymbol{x}, \delta \boldsymbol{v})$, and we must assume that these time scales may overlap with $\tau_{\text {bounce }}$ and $\tau_{\text {pulse }}$. The only separation of time scales that we permit is that all the above processes are assumed to be fast compared to the time scale $\tau_{\text {equil }}$ for global changes in the equilibrium magnetic field geometry and bulk plasma profiles.

Because of the wide range of potentially overlapping time scales, and because the geometry and nonuniformity of the plasma as well as the specific form of the fast ion source are likely to play an important role, the study of such phenomena relies heavily on numerical simulations and their validation against experimental measurements. The validation of the simulation results should be carried out on several levels of detail (e.g., short and long time scales) because the scope of the physics models and the resolution of the phase space covered by the simulations are limited by practical constraints (e.g., computational speed and memory), so it is difficult to ascertain a priori that all relevant physical mechanisms have been included and are simulated with a sufficient degree of realism.

Fortunately, as we will show in this and future papers, the numerical simulations that can be carried out on present-day supercomputers already perform remarkably well for the chirping modes and ALEs that we are interested in. One important factor is that these phenomena are dominated by electromagnetic fluctuations that are localized in the core plasma, have long wavelength (toroidal mode numbers $n=1-3$ ) and frequencies in the band of shear Alfvén waves $\left(f \sim f_{\mathrm{A}}\right)$, so that they fall into the validity regime 

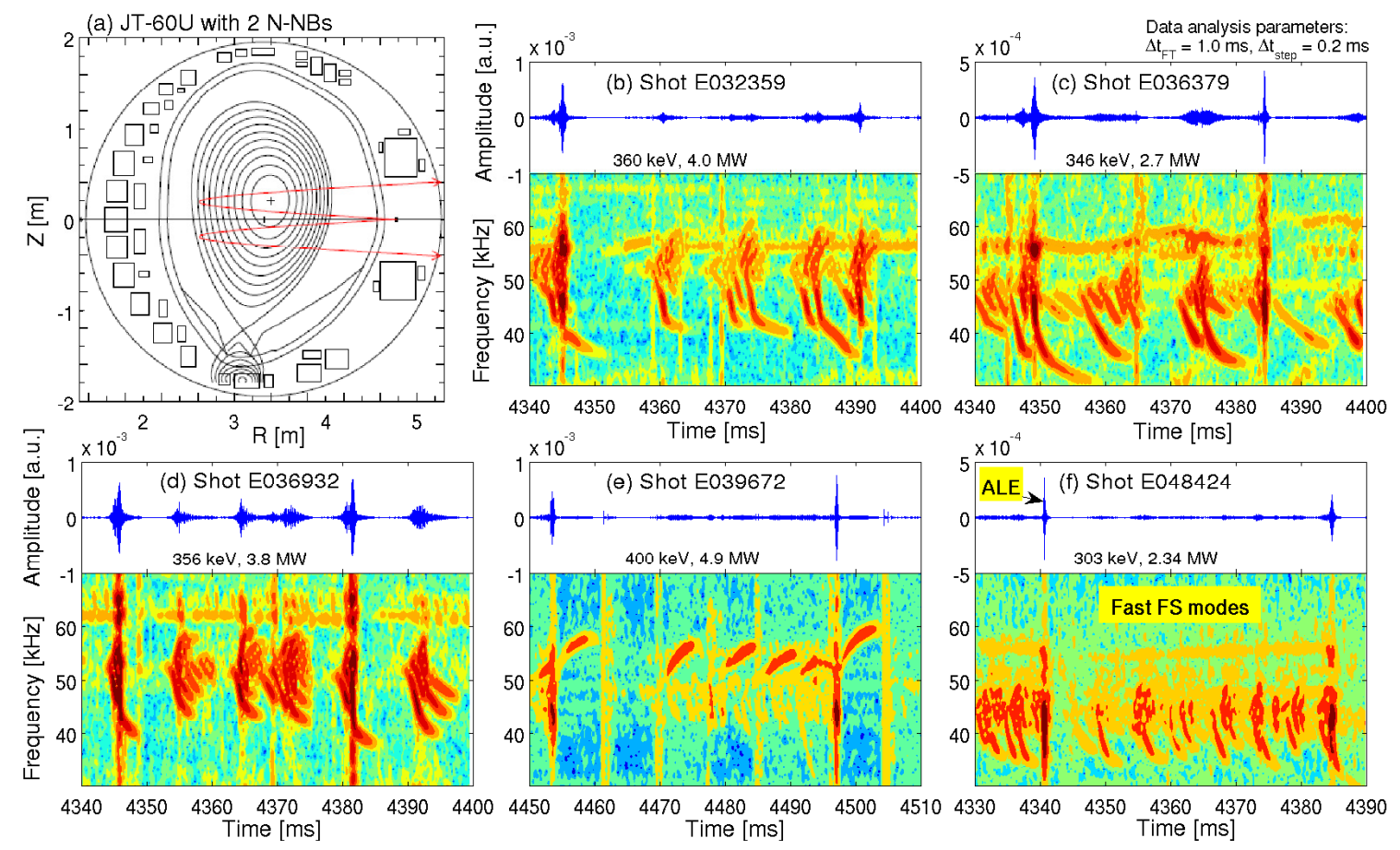

Figure 1. Beam lines of the two negative-ion-based neutral beams (N-NB) in JT-60U (a) and five examples of magnetic signals (b)-(f) taken during the time window $4-5 \mathrm{~s}$, where the safety factor in the plasma core is thought to be in the range $1 \lesssim q \lesssim 2$ in all five shots. Under these conditions, large spikes called "abrupt large events (ALE)" and bursts of chirping fluctuations called "fast frequency-sweeping (fast FS) modes" were routinely observed. A logarithmic color scale is used for the spectrograms in order to show fluctuations with large and small amplitudes in the $30-70 \mathrm{kHz}$ range. In this paper. we reproduce and explain the chirping modes using numerical simulations.

of MHD models. A kinetic treatment is required only for the fast ions, so that an MHD-kinetic hybrid model can be applied [6].

Another factor that raises the probability for the simulations to be both successful and practically relevant, even if carried out only for very few test cases, is the remarkable robustness with which bursts of chirping modes and ALEs occur in JT-60U experiments. These phenomena are routinely observed under a relatively wide range of experimental conditions, as long as the safety factor in the plasma core lies in the range $1 \lesssim q \lesssim 2$, as is also envisioned for ITER and DEMO. Note also that, in JT-60U, these chirps and ALEs occur in the presence of N-NBs injecting deuterons in the range 300-400 keV. Similar MHD fluctuations driven by alpha particles that have slowed down to a few 100 $\mathrm{keV}$ may help to reduce the accumulation of helium ash in the core of a burning plasma.

In the present paper, our goal is to reproduce the bursts of chirping modes seen in N-NB-driven JT-60U plasmas using global nonlinear hybrid simulations performed in realistic geometry, and including realistic fast ion sources and collisions. This distinguishes the present work from similar earlier studies performed with simplified equilibria and fast ion sources $[7,8]$. We use an extended version of the code MEGA $[9,10]$, similar to the one that was recently used for long-time simulations of DIII-D 
tokamak scenarios with positive-ion-based neutral beams (P-NB) [11, 12].

Figure 1 shows the N-NB configuration in JT-60U and five examples of magnetic fluctuation signals exhibiting short intense spikes ("ALEs") interspersed with bursts of chirping modes ("fast FS modes"). In these shots, one can see some common and robust features, such as the frequency range where the fluctuations occur $(30-70 \mathrm{kHz})$, as well as the intervals (5-10 ms) and duration of the bursts of chirping modes (few ms). However, one can also observe differences, such as the dominance of up- or downward chirping, or the coexistence of both. Presumably due to the (slow) evolution of the plasma profiles, the chirping behavior may change during a single experiment on the was (unintentionally) pulsed.

Our simulations can be regarded as successful if they reproduce the common robust features as well as one of the specific chirping behaviors.

Shots E032359-E039672 in Fig. 1(b)-1(e) and Fig. 2 belong to campaigns where the N-NBs were unstable at that time, so attempts were made to produce similar equilibria by tuning, shot by shot, the power of the P-NBs that produce ions with relatively low birth energies of typically $85 \mathrm{keV}$ and less. This led to some differences in the densities and beta values as illustrated in Table 1, and it is likely that the shape of for the different mean frequencies and chirping behavior in Figs. 1 and 2.

Shot E048424 in Fig. 1(f) belongs to a campaign that followed an upgrade of a digitizer for the magnetic sensors (E044000 and after). For instance, the upgrade allowed to increase the sampling frequency for the so-called "saddle loop" coil system from 40 $n=0-4$ of fast-ion-driven modes in the $30-70 \mathrm{kHz}$ range. Compared to Mirnov coils, the saddle loops also offer a better signal-to-noise ratio for low mode numbers, owing to the large surface area of each coil. Results of such measurements are presented in this paper for the first time. that they have shaped the common image of chirping modes. However, since these spectrograms are computed with Fourier transforms over relatively long time windows of about $1 \mathrm{~ms}$, they smear over more rapid dynamics that may be important for constructing an accurate physical picture of the underlying processes. For instance, the fluctuations often occur in short pulses that last only a fraction of a millisecond. Moreover, between many of these pulses, the phase of the oscillations appears to flip abruptly. To our knowledge, this was first recognized in a study of chirping Alfvén modes driven by ion-cyclotron-resonance-heated (ICRH) ions on JET [14]. More recently, similar observations were reported from HL-2A in the presence of P-NB ions [15]. chirps seen on the millisecond time scale in spectrograms such as those in Fig. 1, as well as the pulsations and phase jumps that occur on the time scales of $0.01-0.1 \mathrm{~ms}$. 


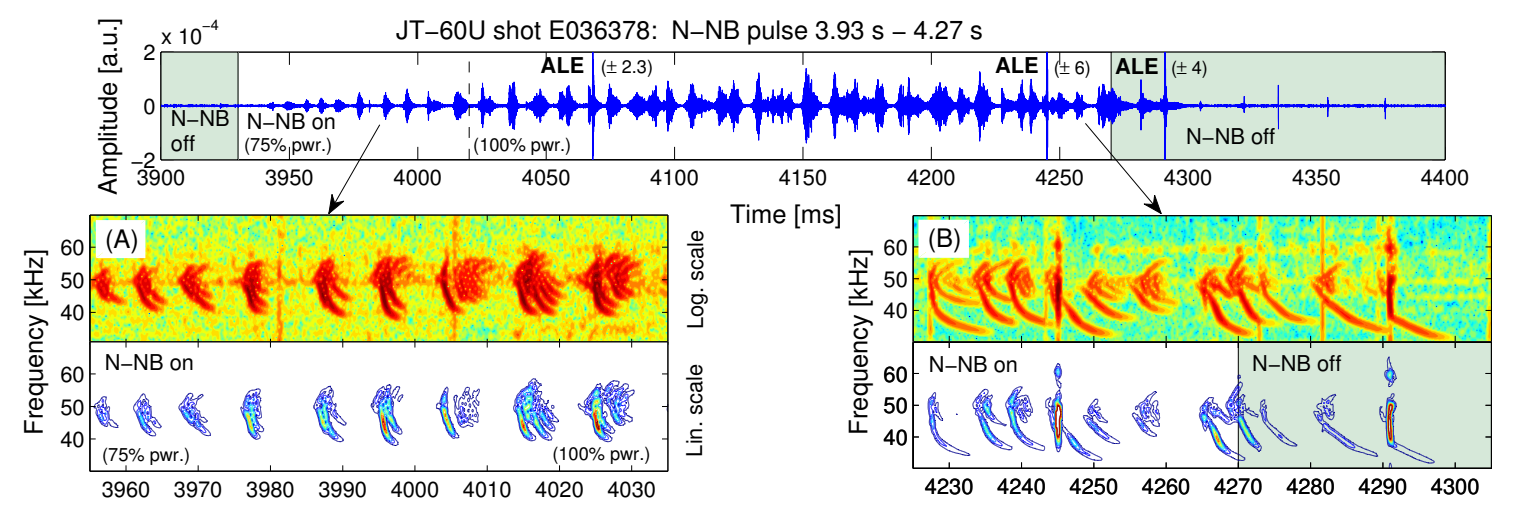

Figure 2. Different chirping patterns seen in JT-60U shot E036378 (A) shortly after the restart of N-NB injection at $t_{\mathrm{start}}=3.93 \mathrm{~s}$, and (B) around the end of the N-NB pulse at $t_{\text {end }}=4.27 \mathrm{~s}$. The N-NBs were off for nearly $200 \mathrm{~ms}$, resumed operation at $t_{\text {start }}=3.93 \mathrm{~s}$ at $75 \%$ of their peak power, and reached $100 \%$ around $t \approx 4.02$, s. Note that the spikes seen at $t>4.3 \mathrm{~s}$, where the beams are off, are not ALEs and do not cause fast ion avalanches.

\begin{tabular}{cccccccc}
\hline Shot & $\begin{array}{c}\text { P-NB } \\
{[\mathrm{MW}]}\end{array}$ & $\begin{array}{c}\mathrm{N}-\mathrm{NB} \\
{[\mathrm{MW}]}\end{array}$ & $\begin{array}{c}E_{0}^{\mathrm{NNB}} \\
{[\mathrm{keV}]}\end{array}$ & $\begin{array}{c}\overline{n_{\mathrm{e}}} \\
10^{19} \mathrm{~m}^{-3}\end{array}$ & $l_{\mathrm{i}}$ & $\begin{array}{c}\langle\beta\rangle \\
{[\%]}\end{array}$ & $\begin{array}{c}\text { Remarks and } \\
\text { references }\end{array}$ \\
\hline E032359 & 0.8 & 4.0 & 360 & 1.1 & 1.2 & 0.52 & $\begin{array}{c}\text { Fig. 5 [1], Figs. 6+10 [2], } \\
\text { Fig. 2 [3], Fig. 1 in [13] }\end{array}$ \\
& & & & & & & N-NB on and off \\
E036378 & 1.31 & 2.64 & 338 & 1.5 & 0.8 & 0.85 & - \\
E036379 & 2.62 & 2.7 & 346 & 1.5 & 0.9 & 1.04 & - \\
E036932 & 1.77 & 3.8 & 356 & 1.2 & 1.0 & 0.98 & Fig. 12(d) in [3] \\
E039672 & 4.2 & 4.9 & 400 & 1.6 & 0.97 & 0.93 & New digitizer (E044000+) \\
E048424 & 1.6 & 2.34 & 303 & 1.3 & 1.2 & 0.75 & Nen \\
\hline
\end{tabular}

Table 1. This table shows how the beam power at the plasma entrance and N-NB birth energy $E_{0}^{\mathrm{NNB}}$ varied between different shots due to experimental constraints, and how this affected the line-averaged electron density $\overline{n_{\mathrm{e}}}$, the internal inductance per unit length $l_{\mathrm{i}}$, and the volume-averaged beta $\langle\beta\rangle$. The values shown are for $t=4 \mathrm{~s}$.

Furthermore, we will show that the pulsations and phase jumps can also be found in the database of N-NB-driven JT-60U experiments, and we will present evidence suggesting that these phenomena can be explained in terms of beat waves that result from the interference between two or more coexisting Alfvénic wave packets.

This paper is organized as follows. In Section 2, we describe the scenario setup, physics model and numerical scheme. The simulation results and comparisons with experimental measurements of chirping modes are presented in Section 3, followed by a discussion of chirping and beating waves in Section 4. A summary, conclusions and outlook to future work are given in Section 5. 


\begin{tabular}{ll}
\hline Location of magnetic axis & $R_{0}=3.395 \mathrm{~m}, Z_{0}=0.204 \mathrm{~m}$ \\
Toroidal field & $B_{0}=1.203 \mathrm{~T}$ \\
Low-field side minor radius & $a_{\mathrm{lfs}}=0.66 \mathrm{~m}$ \\
Alfvén frequency & $f_{\mathrm{A} 0}=209.1 \mathrm{kHz}=v_{\mathrm{A} 0} /\left(2 \pi R_{0}\right)$ \\
Deuterium ion density & $n_{\mathrm{i} 0}=1.731 \times 10^{19} \mathrm{~m}^{-3}$ \\
Plasma current & $I_{\mathrm{p}}=0.571 \mathrm{MA}$ \\
Toroidal beta & $\beta_{0}=3.37 \%$ \\
N-NB power, birth energy & $P_{\mathrm{NNB}}=5 \mathrm{MW}, E_{0}=400 \mathrm{keV}$ \\
\hline
\end{tabular}

Table 2. Plasma and beam parameters for the simulated scenario. The case is based on JT-60U shot E039672 at 4 s [4]. Subscripts "0" indicate values at the magnetic axis.

\section{Scenario setup, physics model and numerical scheme}

In order to be able to achieve our validation and interpretation goals, it is essential that all relevant physical mechanisms are captured by the model equations. At the same time, unnecessary physics overhead should be eliminated in order to make the desired preferable to set up the simulation scenario such that it represents the experimental conditions as closely as possible within the experimental error bars. In this section, we describe how we have set up this scenario and what choices we have made with regard to the physics model in order to reproduce the dynamics of interest.

The toroidal magnetic field $B_{\mathrm{T}}=R \boldsymbol{B} \cdot \boldsymbol{\nabla} \zeta=I / R$ and toroidal current density $J_{\mathrm{T}}=R \boldsymbol{J} \cdot \boldsymbol{\nabla} \zeta=J_{\zeta} / R$ are both positive along the angle $\zeta$. Here, $\zeta$ is chosen to be the reverse of the geometric toroidal angle $\varphi=-\zeta$ of the standard right-handed cylindrical coordinate system $(R, \varphi, Z)$.

25 The magnetic geometry given in terms of the functions $\Psi(R, Z)$ and $I(\Psi)$ was reconstructed by solving the Grad-Shafranov equation using the MHD equilibrium code MEUDAS [16]. MEUDAS requires information about the magnetic coils in JT-60U as 

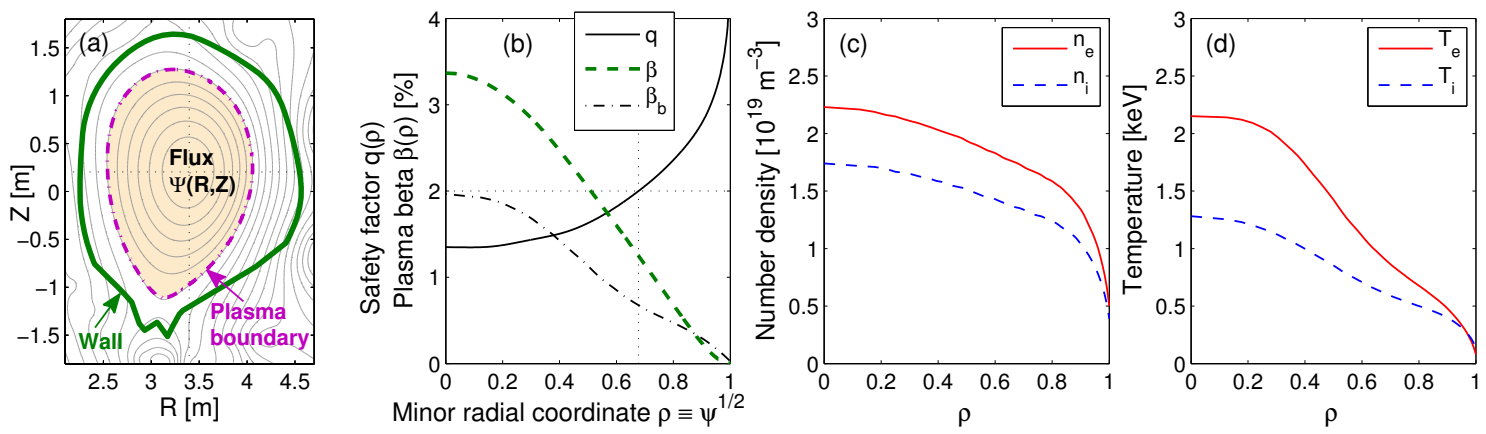

Figure 3. Plasma geometry and profiles based on experimental measurements made in JT-60U shot E039672 at $4 \mathrm{~s}$ [4], which is used as a reference scenario in this work. Panel (a) shows the contours of the equilibrium poloidal flux $\Psi(R, Z)$, the plasma boundary and the wall in a poloidal cross-section of the torus. Panels (b) $-(\mathrm{d})$ show the radial profiles of the safety factor $q(\rho)$, the total toroidal beta $\beta(\rho)$ of the plasma, the number densities $n(\rho)$ and temperatures $T(\rho)$ of electrons ("e") and thermal bulk ions ("i"). We consider this to be a pure deuterium plasma. Note that the shape of the safety factor near the plasma center is uncertain and there may have been a region with negative shear in shot E039672 (cf. Appendix B).

well as estimates for the radial profiles of the safety factor $q(\psi)=\boldsymbol{B} \cdot \boldsymbol{\nabla} \zeta / \boldsymbol{B} \cdot \boldsymbol{\nabla} \vartheta_{\mathrm{f}}$ and plasma pressure $P(\psi)$.

For spatial Fourier analyses of the signals inside the plasma boundary, we use toroidal flux coordinates $\left(\psi, \vartheta_{\mathrm{f}}, \zeta\right)$. Here, the poloidal angle $\vartheta_{\mathrm{f}}$ lies in the poloidal plane, 5 so that it is orthogonal to the toroidal angle $\left(\boldsymbol{\nabla} \vartheta_{\mathrm{f}} \cdot \boldsymbol{\nabla} \zeta=0\right)$. Its increment is given by $\Delta \vartheta_{\mathrm{f}}=\Delta \zeta B_{\mathrm{P}} / B_{\mathrm{T}}$ with $B_{\mathrm{P}}=\boldsymbol{B} \cdot \boldsymbol{\nabla} \vartheta_{\mathrm{f}} /\left|\boldsymbol{\nabla} \vartheta_{\mathrm{f}}\right|$. The normalized flux $0 \leq \psi \leq 1$ serves as a radial-like coordinate defined to be zero at the (magnetic) axis $\ddagger$ and unity at the plasma boundary.

Figure 3(b) shows the profiles of the safety factor $q(\rho)$ and total plasma beta $\beta(\rho) \equiv 2 \mu_{0} P(\rho) / B_{0}^{2}$ as functions of $\rho=\sqrt{\psi}$. The on-axis value of the safety factor is $q_{0} \approx 1.35$ and there is a $q=2$ rational surface at about $2 / 3$ of the plasma radius. The plasma beta has a relatively high on-axis value of $\beta_{0} \approx 3.4 \%$. The estimation of $q(\rho)$ and $\beta(\rho)$ has been a challenging task because we are considering plasmas where high $\beta$ values were achieved by applying strong N-NB drive and by operating with a relatively weak magnetic field strength of $B_{0} \approx 1 \mathrm{~T}$. In such a weak field, motional Stark emission (MSE) measurements do not yield reliable information about the safety factor in the plasma core. Thus, in addition to using global constraints such as the plasma current $I_{\mathrm{p}}$, the estimated shape of the $q$ profile shown in Fig. 3(b) is largely based on MHD spectroscopy as described in Appendix B. The procedure used to estimate the beta profile $\beta(\rho)$ is described in Appendix C.

It is assumed that the background equilibrium defined by $q(\rho)$ and $\beta(\rho)$ remains unchanged during the course of the simulation. This is reasonable because the total pressure will vary by no more than $10 \%$ (even during ALEs) and because the longest

$\ddagger$ Throughout this paper the term "axis" refers to the magnetic axis, where $\nabla \Psi=0$. 
time scale considered is about $50 \mathrm{~ms}$ (a typical ALE period), while $\tau_{\text {equil }}>100 \mathrm{~ms}$.

\subsection{MHD model with fast ion coupling term}

The dynamics of the bulk plasma are described by full MHD equations that are coupled to the effective current density vector of fast ions, $\boldsymbol{j}_{\mathrm{h} \text {,eff }}$, via the momentum balance equation. The form of the equations solved by MEGA $[9,10]$ is as follows:

$$
\begin{aligned}
\partial \rho_{\mathrm{b}} / \partial t= & -\boldsymbol{\nabla} \cdot\left(\rho_{\mathrm{b}} \boldsymbol{u}\right)+\chi \boldsymbol{\nabla}^{2} \rho_{\mathrm{b}}+S_{\rho} \\
\rho_{\mathrm{b}} \partial \boldsymbol{u} / \partial t= & -\rho_{\mathrm{b}} \boldsymbol{u} \cdot \boldsymbol{\nabla} \boldsymbol{u}-\boldsymbol{\nabla} p_{\mathrm{b}}+\left(\boldsymbol{j}-\boldsymbol{j}_{\mathrm{h}, \mathrm{eff}}\right) \times \boldsymbol{B}+\boldsymbol{S}_{u} \\
& -\left[\boldsymbol{\nabla} \times\left(\nu \rho_{\mathrm{b}} \boldsymbol{\nabla} \times \boldsymbol{u}\right)+\frac{4}{3} \boldsymbol{\nabla}\left(\nu \rho_{\mathrm{b}} \boldsymbol{\nabla} \cdot \boldsymbol{u}\right)\right], \\
\partial \boldsymbol{B} / \partial t= & -\boldsymbol{\nabla} \times \boldsymbol{E}, \\
\boldsymbol{E} \quad & -\boldsymbol{u} \times \boldsymbol{B}+\eta \boldsymbol{j}+\boldsymbol{S}_{E}, \\
\mu_{0} \boldsymbol{j}= & \boldsymbol{\nabla} \times \boldsymbol{B}, \\
\partial p_{\mathrm{b}} / \partial t= & -\boldsymbol{\nabla} \cdot\left(p_{\mathrm{b}} \boldsymbol{u}\right)-(\Gamma-1) p_{\mathrm{b}} \boldsymbol{\nabla} \cdot \boldsymbol{u}+\chi \boldsymbol{\nabla}^{2} p_{\mathrm{b}} \\
& +\nu \rho_{\mathrm{b}}(\Gamma-1)\left[(\boldsymbol{\nabla} \times \boldsymbol{u})^{2}+\frac{4}{3}(\boldsymbol{\nabla} \cdot \boldsymbol{u})^{2}\right] \\
& +(\Gamma-1)\left(\boldsymbol{j}-\boldsymbol{j}_{\mathrm{h}, \mathrm{eff}}\right) \cdot\left(\eta \boldsymbol{j}+S_{E}\right)+S_{p} .
\end{aligned}
$$

Here, $\rho_{\mathrm{b}}$ and $p_{\mathrm{b}}$ are the density and scalar pressure of the bulk plasma, $\boldsymbol{u}$ is the singlefluid MHD velocity vector, $\boldsymbol{B}$ and $\boldsymbol{E}$ are the magnetic and electric field vectors, and

$\boldsymbol{j}$ is the total current density of the plasma, including the fast ion component. Since equilibrium flows are ignored, $\boldsymbol{E}=\delta \boldsymbol{E}(t)$ and $\boldsymbol{u}=\delta \boldsymbol{u}(t)$ consist only of fluctuations and a fluctuating component; for instance, $\boldsymbol{j}(t)=\boldsymbol{j}_{\mathrm{eq}}+\delta \boldsymbol{j}(t)$. The source terms

$$
\begin{aligned}
& S_{\rho}=-\chi \boldsymbol{\nabla}^{2} \rho_{\mathrm{b}, \mathrm{eq}}, \\
& \boldsymbol{S}_{u}=\boldsymbol{\nabla} p_{\mathrm{b}, \mathrm{eq}}-\left(\boldsymbol{j}_{\mathrm{eq}}-\boldsymbol{j}_{\mathrm{h}, \mathrm{eq}}\right) \times \boldsymbol{B}_{\mathrm{eq}}, \\
& \boldsymbol{S}_{E}=-\eta \boldsymbol{j}_{\mathrm{eq}} \\
& S_{p}=-\chi \boldsymbol{\nabla}^{2} p_{\mathrm{b}, \mathrm{eq}},
\end{aligned}
$$

are used to enforce equilibrium MHD force balance and compensate the diffusion and dissipation of the equilibrium fields $\rho_{\mathrm{b}, \mathrm{eq}}, p_{\mathrm{b}, \mathrm{eq}}, \boldsymbol{j}_{\mathrm{eq}}$ and $\boldsymbol{B}_{\text {eq }}$. The energy dissipated by resistive and viscous diffusion is converted into heat in the thermodynamic equation of state (8) for the bulk pressure $p_{\mathrm{b}}$.

The values of the electric resistivity $\eta$, viscosity $\nu$ and thermal diffusivity $\chi$ are fixed at $\mu_{0} \eta=\nu=\chi=10^{-6} v_{\mathrm{A} 0} R_{0}$. Compressibility is controlled by the specific heat ratio, which is fixed at $\Gamma=5 / 3$. For a justification of these values, see our recent sensitivity study in Ref. [17]. There, it was also noted that the primary role of the diffusion coefficients is to realize MHD closure by dissipating small-scale structures whose evolution is not described by the MHD model in a physical way. Moreover, diffusion controlled by $\chi$ is also used to dissipate unphysical artifacts that may develop 
in the advected scalar fields $\rho_{\mathrm{b}}$ and $p_{\mathrm{b}}$ in the course of long-time simulations due to the absence of parallel streaming of particles in the MHD model.

The effective current density of fast ion guiding centers, $\boldsymbol{j}_{\mathrm{h}, \mathrm{eff}}=\boldsymbol{j}_{\mathrm{d} \text {,eff }}+\boldsymbol{j}_{\mathrm{mag}}$, consists of drift and magnetization currents, which are computed from the guiding center 5 distribution $F_{\mathrm{h}}$ as

$$
\boldsymbol{j}_{\mathrm{d}, \mathrm{eff}}=\int \mathrm{d}^{3} v F_{\mathrm{h}} q_{\mathrm{h}}\left(\boldsymbol{U}_{\mathrm{gc}}-\boldsymbol{v}_{E}^{*}\right), \quad \boldsymbol{j}_{\mathrm{mag}}=-\boldsymbol{\nabla} \times \int \mathrm{d}^{3} v F_{\mathrm{h}} \mu \hat{\boldsymbol{b}}
$$

where $\hat{\boldsymbol{b}}=\boldsymbol{B} / B$ is the unit vector along the magnetic field, $\mu=m_{\mathrm{h}} v_{\perp}^{2} /(2 B)$ is the magnetic moment, $q_{\mathrm{h}}$ the electric charge, and $\boldsymbol{U}_{\mathrm{gc}}$ is the guiding center velocity defined in Eqs. (14)-(17) below. By subtracting $\boldsymbol{v}_{E}^{*}$ from the integrand of $\boldsymbol{j}_{\text {d,eff }}$ in Eq. (13), we enforce the exact quasineutral cancellation of the $\boldsymbol{E} \times \boldsymbol{B}$ drift and ignore the correction $\left(1-B^{*} / B\right) \boldsymbol{v}_{E}$, which is small for fast ions.S

The simulation domain is a rectangular torus set around the wall as shown in Fig. 3(a), and it is discretized in cylinder coordinates $(R, \varphi, Z)$, where $\varphi=-\zeta$. The MHD equations (3)-(8) are solved inside this domain using standard 4th-order RungeKutta and finite difference schemes, while suppressing velocity fluctuations $\delta \boldsymbol{u}$ at and beyond the plasma boundary, which is shown as a dash-dotted line in Fig. 3(a). This boundary condition suppresses external modes. In order to avoid possible numerical problems at the magnetic X-point of the JT-60U divertor configuration, the plasma boundary is defined to lie at $98 \%$ of the flux space within the separatrix.

Following the analyses presented in Refs. $[17,18]$, the cylinder coordinates $(R, \varphi, Z)$ are discretized using $\left(N_{R}, N_{\varphi}, N_{Z}\right)=(384,96,352)$ grid points, which is sufficient to simulate long-wavelength fluctuations with toroidal mode numbers $n=0-4$. Parallelization is performed through spatial domain decomposition. The self-consistent long-time simulations were run using $4096 \mathrm{MPI}$ processes (16 in each spatial dimension). The time step for the MHD module is $\Delta t_{\mathrm{mhd}} \times \omega_{\mathrm{ci}}=0.05$ in units of the deuteron Larmor frequency $\omega_{\mathrm{ci}}=e B_{0} / m_{\mathrm{D}}$. This corresponds to $\Delta t_{\mathrm{mhd}} \times \omega_{\mathrm{A} 0} \approx 1.1 \times 10^{-3}$ Alfvén times $(\approx 1 \mathrm{~ns})$, with $\omega_{\mathrm{A} 0} \equiv v_{\mathrm{A} 0} / R_{0}=2 \pi \times 209.1 \mathrm{kHz}$.

\subsection{Guiding center equations of motion}

The fast ion phase space is sampled by simulation particles, which follow the orbits of guiding centers as prescribed by the following equations of motion [19]:

$$
\begin{aligned}
& \dot{\boldsymbol{R}}_{\mathrm{gc}}=\left(\boldsymbol{v}_{\|}^{*}+\boldsymbol{v}_{E}^{*}+\boldsymbol{v}_{B}\right) \equiv \boldsymbol{U}_{\mathrm{gc}}, \\
& m_{\mathrm{h}} v_{\|} \dot{v}_{\|}=\boldsymbol{v}_{\|}^{*} \cdot\left(q_{\mathrm{h}} \boldsymbol{E}-\mu \boldsymbol{\nabla} B\right)
\end{aligned}
$$

with

$$
\rho_{\|} \equiv \frac{m_{\mathrm{h}} v_{\|}}{q_{\mathrm{h}} B}, \quad B^{*} \equiv B\left[1+\rho_{\|} \hat{\boldsymbol{b}} \cdot(\boldsymbol{\nabla} \times \hat{\boldsymbol{b}})\right], \quad \boldsymbol{v}_{E}^{*}=\frac{\boldsymbol{E} \times \hat{\boldsymbol{b}}}{B^{*}},
$$

$\mathcal{S}$ Since fast ions move at Alfvénic velocities, $v_{\|} / v_{\mathrm{A} 0} \sim 1$, the ratio of the term $\left(1-B^{*} / B\right) \boldsymbol{v}_{E}$ to the curvature drift contained in $\boldsymbol{v}_{\|}^{*}$ is of order $\mathcal{O}\left(a / R_{0} \times v_{E} / v_{\|}\right) \sim\left(10^{-3} \ldots 10^{-2}\right)$, even during an ALE, where the electric drift reaches a magnitude of $v_{E \vartheta} / v_{\mathrm{A} 0} \sim 10^{-2}$ for a short interval of about $0.1 \mathrm{~ms}$. 

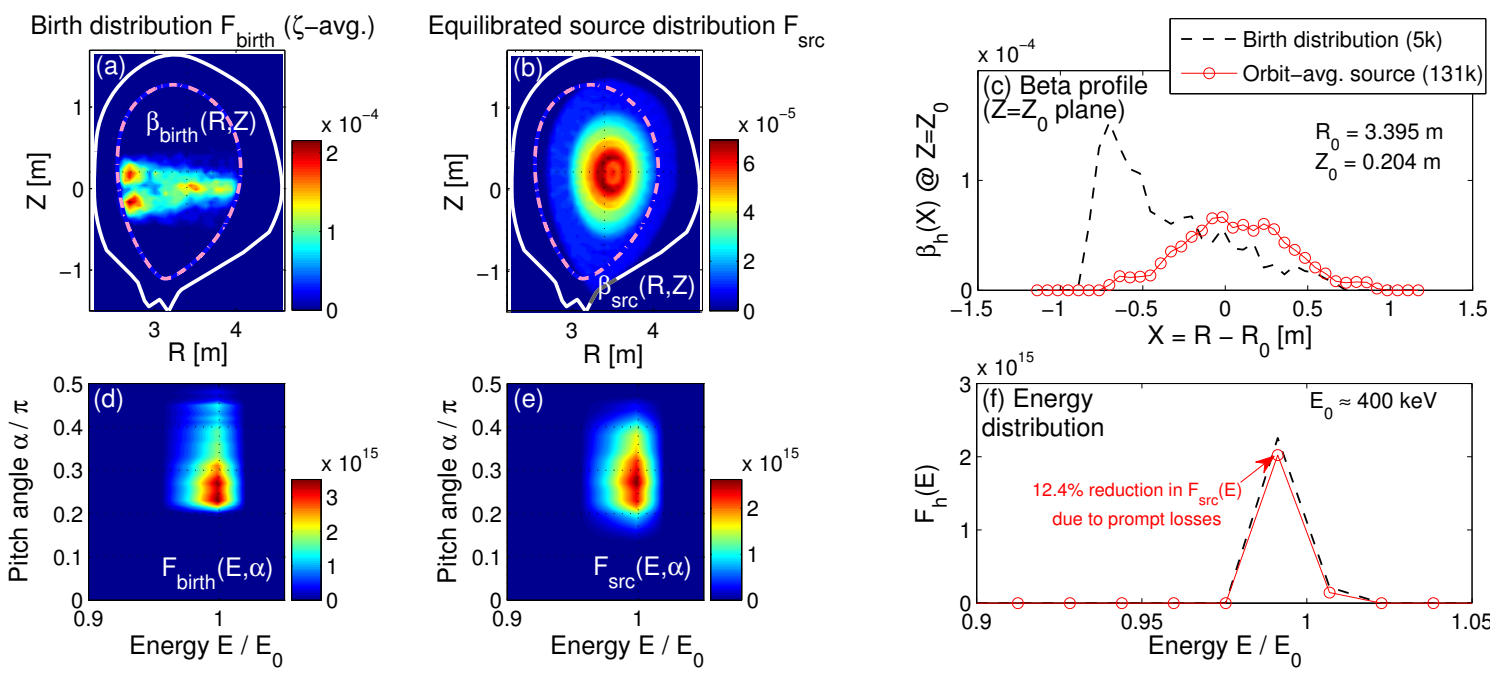

Figure 4. N-NB ion birth distribution $F_{\text {birth }}$ used in OFMC simulations (a,d), and the corresponding equilibrated source distribution $F_{\text {src }}$ used in MEGA simulations (b,e). $F_{\text {birth }}$ is represented by $N_{\text {birth }}=5 \mathrm{k}$ test particles. $F_{\text {src }}$ is represented by $N_{\text {src }}=131 \mathrm{k}$ simulation particles, which are distributed along the unperturbed guiding center orbits that start from the birth points given by $F_{\text {birth }}$. (a)-(c): Beta fields $\beta(R, Z)$ and their radial profiles $\beta(X)$ evaluated at $Z=Z_{0}$ and plotted as functions of $X=R-R_{0}$. (d)-(f): Spatially averaged velocity distributions $F(E, \alpha)$ and $F(E)$.

$$
\boldsymbol{v}_{\|}^{*}=\frac{v_{\|}}{B^{*}}\left(\boldsymbol{B}+\rho_{\|} B \boldsymbol{\nabla} \times \hat{\boldsymbol{b}}\right), \quad \boldsymbol{v}_{B}=-\frac{\mu}{q_{\mathrm{h}} B^{*}} \boldsymbol{\nabla} B \times \hat{\boldsymbol{b}}
$$

where the magnetic moment is adiabatically conserved: $\dot{\mu} \equiv \mathrm{d} \mu / \mathrm{d} t=0$. Using the standard 4th-order Runge-Kutta scheme, the particles are pushed with time steps of size $\Delta t_{\text {push }} \times \omega_{\text {ci }}=0.20$, which is four times larger than the MHD time step. The simulation particles are allowed to exit the plasma and traverse the vacuum region as 5 shown in Fig. 2(a) of Ref. [20]. Particles that collide with the wall are considered lost.

The evolution of the fast ion guiding center distribution $F_{\mathrm{h}}(\boldsymbol{Z}, t)$ is represented with the full- $f$ method, and the first-order particle-in-cell (PIC) method is used to map the field values to the particle positions and the particle weights to the spatial grid. Finite Larmor radius (FLR) effects are taken into account using $N_{\text {gyro }}=4$ satellite particles that are placed around the guiding center positions as illustrated in Fig. 1 of Ref. [17]. Electromagnetic forces acting on the simulation particles are computed by averaging the field values at the satellite positions. The fast ion current density $\boldsymbol{j}_{\mathrm{h} \text {,eff }}$ is computed by distributing the weights of simulation particles equally to all satellites and, from there, mapping the weights to the spatial grid. After that, a Fourier filter is applied to $\boldsymbol{j}_{\mathrm{h}, \mathrm{eff}}(R, \varphi, Z)$ along the toroidal angle, keeping only the harmonics $n=1,2,3$.

\subsection{Fast ion source}

The fast ions are born along the two beam lines shown in Fig. 1(a). Their birth distribution $F_{\text {birth }}\left(R, Z, \zeta, E, v_{\|}\right)$was computed using the ionization model described in Ref. [21], which was implemented in the code OFMC [22, 23]. Figure 4(a) shows its 
projection into the poloidal $(R, Z)$ plane in the form of the toroidally averaged beta field

$\beta_{\text {birth }}(R, Z)=\int \frac{\mathrm{d} \zeta R_{0}}{2 \pi R} \int \frac{\mathrm{d}^{3} v}{n_{\mathrm{i} 0}} F_{\text {birth }} \frac{E}{m_{\mathrm{h}} v_{\mathrm{A} 0}^{2}}$. Its radial profile $\beta_{\mathrm{birth}}(X)$ at the height of the magnetic axis, $Z=Z_{0}=0.204 \mathrm{~m}$, is shown in Fig. 4(c) as a function of $X=R-R_{0}$. Figure $4(\mathrm{~d})$ shows the spatially averaged velocity distribution $F_{\text {birth }}(E, \alpha)$. The kinetic 5 energy $E=m_{\mathrm{h}} v^{2} / 2$ is normalized by the nominal birth energy $E_{0}=m_{\mathrm{h}} v_{0}^{2} / 2=400 \mathrm{keV}$, so that there is a sharp peak around $E / E_{0}=1$. Along the pitch angle $\alpha \equiv \sin ^{-1}\left(v_{\|} / v\right)$ the birth distribution peaks near $\alpha / \pi \approx 0.27\left(v_{\|} / v \approx 0.75\right)$.

For historical reasons, we do not use $F_{\text {birth }}$ in MEGA directly but assume that the fast ion birth distribution will have time to fully equilibrate before it is modified by collisions. In other words, we assume that the fast ions will spread out along their unperturbed guiding center orbits before undergoing significant collisional slowdown or scattering. This equilibrated source distribution $F_{\text {src }}$ is obtained from $F_{\text {birth }}$ by applying the orbit-based particle loading scheme described in Ref. [24]. The birth distribution $F_{\text {birth }}$, which consists of $N_{\text {birth }}=5 \mathrm{k}$ samples, provides the initial positions for unperturbed guiding center orbits. 622 orbits were discarded because they intersect the wall (so-called "prompt loss"). Each of the remaining orbits is sampled by 30 simulation particles that are distributed along the orbit contour at spatial intervals that correspond to equal intervals in time (cf. Eq. (30) in Ref. [24]). This gave a 4-d source distribution $F_{\text {src }}\left(R, Z, E, v_{\|}\right)$that is sampled by $N_{\text {src }}=131 \mathrm{k}$ particles and whose 2 -d projections $\beta_{\mathrm{src}}(R, Z)$ and $F_{\mathrm{src}}(E, \alpha)$ are shown in Figs. 4(b) and 4(e).

In Figs. $4(\mathrm{c})$ and $4(\mathrm{f})$, the 1-d projections $\beta_{\mathrm{src}}(X)$ and $F_{\mathrm{src}}(E)$ (pitch-angle average) of the equilibrated source distribution used in MEGA are compared to those of the non-equilibrium birth distribution $F_{\text {birth }}$ used in OFMC. The difference seen between the energy distributions $F_{\text {birth }}(E)$ and $F_{\text {src }}(E)$ in Fig. $4(\mathrm{f})$ amounts to $12.4 \%$, which is 25 precisely the fraction of particles in $F_{\text {birth }}$ that would be promptly lost in the absence of collisions.

At $t=0$, the simulation starts without any fast ions. At each injection time step $\Delta t_{\text {inj }}$, a certain number of new simulation particle is launched with initial guiding center coordinates $\boldsymbol{Z}_{\mathrm{gc}}=\left(R, \varphi, Z, E, v_{\|}\right)$that are sampled randomly from the 4 -d source distribution $F_{\mathrm{src}}\left(R, Z, E, v_{\|}\right)$and the toroidal angle $0 \leq \varphi<2 \pi$. In the selfconsistent MEGA simulation that includes MHD, sources and collisions, we inject one new simulation particle per pushing time step, $\Delta t_{\text {inj }}=\Delta t_{\text {push }}$, which gives $0.3 \mathrm{M}$ particles per millisecond of physical time. During the $35 \mathrm{~ms}$ time interval examined in this paper, about $10.5 \mathrm{M}$ particles are injected and $9.8 \mathrm{M}$ of them are still confined at the end of the simulation. "Classical" MEGA simulations, which do not include MHD fluctuations, are run with fewer particles and larger time steps.

\subsection{Fast ion collisions and sink}

In MEGA simulations reported in earlier works by Todo et al. $[8,11,12]$, who performed long-time simulations for TFTR and DIII-D tokamak scenarios, a Monte Carlo collision model formulated by Boozer \& Kuo-Petravic [25] was used, which describes the slow- 


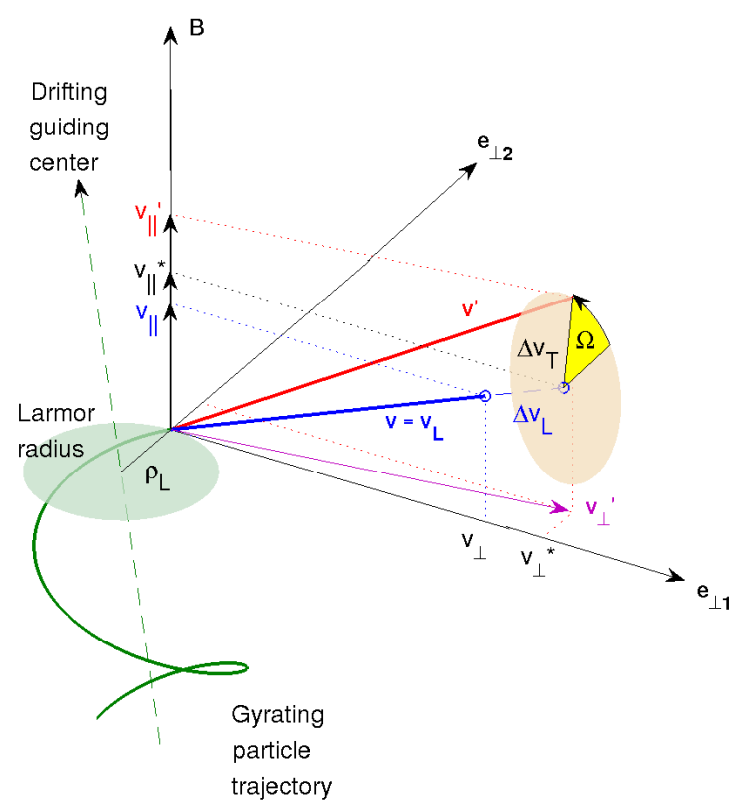

Figure 5. Schematic illustration of the collision model given by Eqs. (18)-(23).

down and pitch-angle scattering processes explicitly as a gradual reduction of velocity $v=\sqrt{2 E / m_{\mathrm{h}}}$ and a random diffusion along the pitch angle coordinate $\Lambda=v_{\perp} / v$.

In the present work, we adopt the collision model used in the OFMC code [22]. As shown in Fig. 5, the model describes in a very elementary way how, during a time

step $\Delta t_{\text {coll }}$, the velocity vector components of a fast ion change from $\boldsymbol{v}=\left(v_{\|}, v_{\perp}\right)$ to new values $\boldsymbol{v}^{\prime}=\left(v_{\|}^{\prime}, v_{\perp}^{\prime}\right)$ due to collisions with thermal bulk ions and electrons. Using elementary geometric rules, Fig. 5 can be expressed mathematically as

$$
\begin{aligned}
& \left(v^{\prime}\right)^{2}=\left(v_{\mathrm{L}}+\Delta v_{\mathrm{L}}\right)^{2}+\Delta v_{\mathrm{T}}^{2}, \quad\left(v_{\perp}^{\prime}\right)^{2}=\left(v^{\prime}\right)^{2}-\left(v_{\|}^{\prime}\right)^{2}, \\
& \frac{v_{\|}^{*}}{v_{\mathrm{L}}+\Delta v_{\mathrm{L}}}=\frac{v_{\|}}{v_{\mathrm{L}}}, \quad \frac{v_{\|}^{\prime}-v_{\|}^{*}}{\Delta v_{\mathrm{T}} \sin \Omega}=\frac{v_{\perp}}{v_{\mathrm{L}}},
\end{aligned}
$$

which can be transformed into the following equations for the new velocity components:

$$
\begin{aligned}
& v_{\|}^{\prime}=\frac{v_{\|}}{v}\left(v+\Delta v_{\mathrm{L}}\right)+\frac{v_{\perp}}{v} \Delta v_{\mathrm{T}} \sin \Omega, \\
& v_{\perp}^{\prime}=\sqrt{\left(v_{\mathrm{L}}+\Delta v_{\mathrm{L}}\right)^{2}+\Delta v_{\mathrm{T}}^{2}-\left(v_{\|}^{\prime}\right)^{2}} .
\end{aligned}
$$

The subscripts " $\mathrm{L}$ " and "T" indicate components that are longitudinal and transverse to the initial velocity vector $\boldsymbol{v}$, so that $v \equiv|\boldsymbol{v}|=v_{\mathrm{L}}$. The deflection angle $\Omega$ in the transverse plane is a random variable sampled uniformly from the interval $0 \leq \Omega<2 \pi$. The quantities $\Delta v_{\mathrm{L}}$ and $\Delta v_{\mathrm{T}}$ are random variables with Gaussian distributions. Their mean values $\langle\Delta v\rangle$ and standard deviations $\left\langle\widetilde{v}^{2}\right\rangle \equiv\left\langle(\Delta v-\langle\Delta v\rangle)^{2}\right\rangle$ are computed using formulas taken from the textbook by Trubnikov [26]: for "test particles (t)" (i.e., fast ions) colliding with Maxwellian "plasma particles (p)" (i.e., bulk ions and electrons), 
we have $\left\langle\Delta v_{\mathrm{T}}\right\rangle_{\mathrm{p}}=0$ and

$$
\begin{aligned}
& \frac{\left\langle\Delta v_{\mathrm{L}}\right\rangle_{\mathrm{p}}}{\Delta t_{\mathrm{coll}} v_{\mathrm{A} 0}^{2} / R_{0}}=C_{\mathrm{p}} \frac{\hat{n}_{\mathrm{p}}\left(\psi_{\mathrm{t}}\right)}{\hat{T}_{\mathrm{p}}\left(\psi_{\mathrm{t}}\right)} \frac{\mu\left(\chi_{\mathrm{p}}\left(v_{\mathrm{t}}\right)\right)}{2 \chi_{\mathrm{p}}\left(v_{\mathrm{t}}\right)}, \\
& \frac{\left\langle\Delta \widetilde{v}_{\mathrm{L}}^{2}\right\rangle_{\mathrm{p}}}{\Delta t_{\mathrm{coll}} v_{\mathrm{A} 0}^{3} / R_{0}}=D_{\mathrm{p}} \frac{\hat{n}_{\mathrm{p}}\left(\psi_{\mathrm{t}}\right)}{\hat{T}_{\mathrm{p}}^{1 / 2}\left(\psi_{\mathrm{t}}\right)} \frac{\mu\left(\chi_{\mathrm{p}}\left(v_{\mathrm{t}}\right)\right)}{2 \chi_{\mathrm{p}}^{3 / 2}\left(v_{\mathrm{t}}\right)}, \\
& \frac{\left\langle\Delta \widetilde{v}_{\mathrm{T}}^{2}\right\rangle_{\mathrm{p}}}{\Delta t_{\mathrm{coll}} v_{\mathrm{A} 0}^{3} / R_{0}}=D_{\mathrm{p}} \frac{\hat{n}_{\mathrm{p}}\left(\psi_{\mathrm{t}}\right)}{\hat{T}_{\mathrm{p}}^{1 / 2}\left(\psi_{\mathrm{t}}\right)}\left[\frac{\mu\left(\chi_{\mathrm{p}}\left(v_{\mathrm{t}}\right)\right)+\mu^{\prime}\left(\chi_{\mathrm{p}}\left(v_{\mathrm{t}}\right)\right)}{\chi_{\mathrm{p}}^{1 / 2}\left(v_{\mathrm{t}}\right)}-\frac{\mu\left(\chi_{\mathrm{p}}\left(v_{\mathrm{t}}\right)\right)}{2 \chi_{\mathrm{p}}^{3 / 2}\left(v_{\mathrm{t}}\right)}\right] .
\end{aligned}
$$

The function $\mu(\chi)$ and its argument are defined as

$\mu(\chi)=\operatorname{Erf}(\sqrt{\chi})-2 \sqrt{\frac{\chi}{\pi}} \exp (-\chi), \quad \mu^{\prime} \equiv \frac{\mathrm{d} \mu}{\mathrm{d} \chi}, \quad \chi_{\mathrm{p}} \equiv \frac{m_{\mathrm{p}} v_{\mathrm{t}}^{2}}{2 T_{\mathrm{p}}}=\frac{v_{\mathrm{t}}^{2}}{v_{\mathrm{p}}^{2}}$.

The scalar coefficients are defined as

$$
C_{\mathrm{p}} \equiv \frac{v_{\beta}}{v_{\mathrm{A} 0}} \frac{3 \sqrt{\pi}}{2} \frac{M_{\mathrm{t}}}{\hat{\tau}_{\mathrm{s} 0}} Z_{\mathrm{p}}^{2}\left(\frac{m_{\mathrm{p}}}{m_{\mathrm{t}}}+1\right), \quad D_{\mathrm{p}} \equiv \frac{v_{\beta}^{2}}{v_{\mathrm{A} 0}^{2}} \frac{3 \sqrt{\pi}}{2} \frac{M_{\mathrm{t}}}{\hat{\tau}_{\mathrm{s} 0}} Z_{\mathrm{p}}^{2} \sqrt{\frac{m_{\mathrm{p}}}{m_{\mathrm{t}}}},
$$

5 with

$$
v_{\beta}^{2} \equiv \frac{2 T_{\mathrm{e} 0}}{m_{\mathrm{t}}}, \quad M_{\mathrm{t}} \equiv\left(\frac{m_{\mathrm{t}}}{m_{\mathrm{e}}}\right)^{3 / 2}\left(1+\frac{m_{\mathrm{t}}}{m_{\mathrm{e}}}\right)^{-1},
$$

The slowing down time is computed as

$$
\hat{\tau}_{\mathrm{s} 0}=6.3 \times 10^{14} \times \frac{A_{\mathrm{t}}\left(T_{\mathrm{e} 0}[\mathrm{keV}]\right)^{3 / 2}}{Z_{\mathrm{t}}^{2} \ln \Lambda_{\mathrm{e} 0} n_{\mathrm{e} 0}\left[\mathrm{~m}^{-3}\right]} \frac{v_{\mathrm{A} 0}}{R_{0}} \frac{R_{0}}{\rho_{\mathrm{A} 0}} .
$$

with mass number $A_{\mathrm{t}} \equiv m_{\mathrm{t}} / m_{\mathrm{D}}$, charge number $Z_{\mathrm{t}} \equiv q_{\mathrm{t}} / e$ and Column logarithm $\ln \Lambda_{\mathrm{e} 0}=16$.

The longitudinal slow-down $\left\langle\Delta v_{\mathrm{L}}\right\rangle$ dominates at high energies $E>E_{\text {crit }}$ due to collisional drag from electrons. Ignoring impurities, the critical energy is approximately $E_{\text {crit }} \approx 14.8 \times T_{\mathrm{e}}[\mathrm{keV}] \lesssim 30 \mathrm{keV}$ (cf. Eq. (15) in Ref. [27]). Transverse diffusion $\left\langle\Delta v_{\mathrm{T}}^{2}\right\rangle$ occurs mainly in the form of pitch-angle scattering through collisions with bulk ions, and it plays an important role at lower energies, $E \lesssim E_{\text {crit }}$. Finally, $\left\langle\Delta v_{\mathrm{L}}^{2}\right\rangle$ captures the longitudinal part of energy diffusion, which is weak in the cases considered here.

If the collision operation is applied at the position of a satellite particle, one obtains a spatial diffusion effect that is associated with the nonuniform collisionality across the diameter of a fast ion Larmor orbit. This may play a role at energies below $100 \mathrm{keV}$, where pitch-angle scattering has a significant effect; although, the smaller Larmor radius may compensate it at least partially. In the high energy range of interest here, where collisional slow-down is dominant, the spatial diffusion due to fast ion FLR was found to cause a negligibly small difference: $\Delta \beta_{\mathrm{h}}(\rho) / \beta_{\mathrm{h}}(\rho)$ is within $2 \%$ after $100 \mathrm{~ms}$. Thus, in the present work, collisions are simply evaluated at the guiding center position, so that spatial diffusion arises only through changes in the magnetic drift orbits caused by $\Delta v_{\mathrm{L}}$ and $\Delta v_{\mathrm{T}}$.

In the MEGA simulations reported here, collisions are simulated at every particle pushing time step, $\Delta t_{\text {coll }}=\Delta t_{\text {push }}$. Particles that slow down below $E_{\min } / E_{0}=0.213(85$ 
$\mathrm{keV}$ ) are discarded. Below this energy, fast ions originating from P-NBs were present in the JT-60U experiments, but they were not included in the source model $F_{\text {src }}$ used here because they are not expected to interact resonantly with the modes of interest.

\section{Simulation results and comparison with experiments}

5 $F_{\text {src }}$ described in Section 2.4, MEGA can be operated as a "classical" orbit-following Monte-Carlo code when the MHD solver is turned off. When the MHD solver is turned on along with sources and collisions, we speak of a "self-consistent" simulation, where only the background equilibrium and plasma profiles are held fixed.

10 first $35 \mathrm{~ms}$ of physical time after the start of beam injection. On the supercomputer Helios, the classical simulation of $35 \mathrm{~ms}$ accumulating $4.9 \mathrm{M}$ particles took 28 hours on 256 cores. The self-consistent simulation accumulating $9.8 \mathrm{M}$ particles took 39 days using 4096 cores. Preliminary results obtained with fewer particles and simulating the course of the last three years [28, 29]. The results of the self-consistent MEGA simulations reported here were obtained with 4 times more simulation particles and include fluctuations with toroidal mode numbers $n=1-4$, where $n=1-3$ are driven by fast ions. The essential features of the earlier preliminary results are reproduced, so we consider them to be numerically robust.

First, in Section 3.1, we examine the collisional slow-down and transport of fast ions. After benchmarking the results of a classical MEGA simulation against OFMC results, we evaluate the fast ion transport caused by MHD activity in the self-consistent MEGA simulation. self-consistent MEGA simulation with JT-60U experiments. The fundamental type of shear Alfvén mode responsible for the chirping phenomena is identified in Section 3.2 and its nonlinear dynamics are examined in Sections 3.3 and 3.4.

We will compare our simulation results with experimental data from two JT-60U experiments. One shot is E036378 after $t=3.93 \mathrm{~s}$ (cf. Fig. 2), where the beams resumed operation after having been off for nearly $200 \mathrm{~ms}$. The other shot is E048424, where we consider the dynamics following an ALE at $t=4.34 \mathrm{~s}$ (cf. Fig. 1(f)). A significant portion of fast ions has been convected out during the ALE, and the beams have begun to restore the fast ion population in the core plasma. These kinds of conditions are 

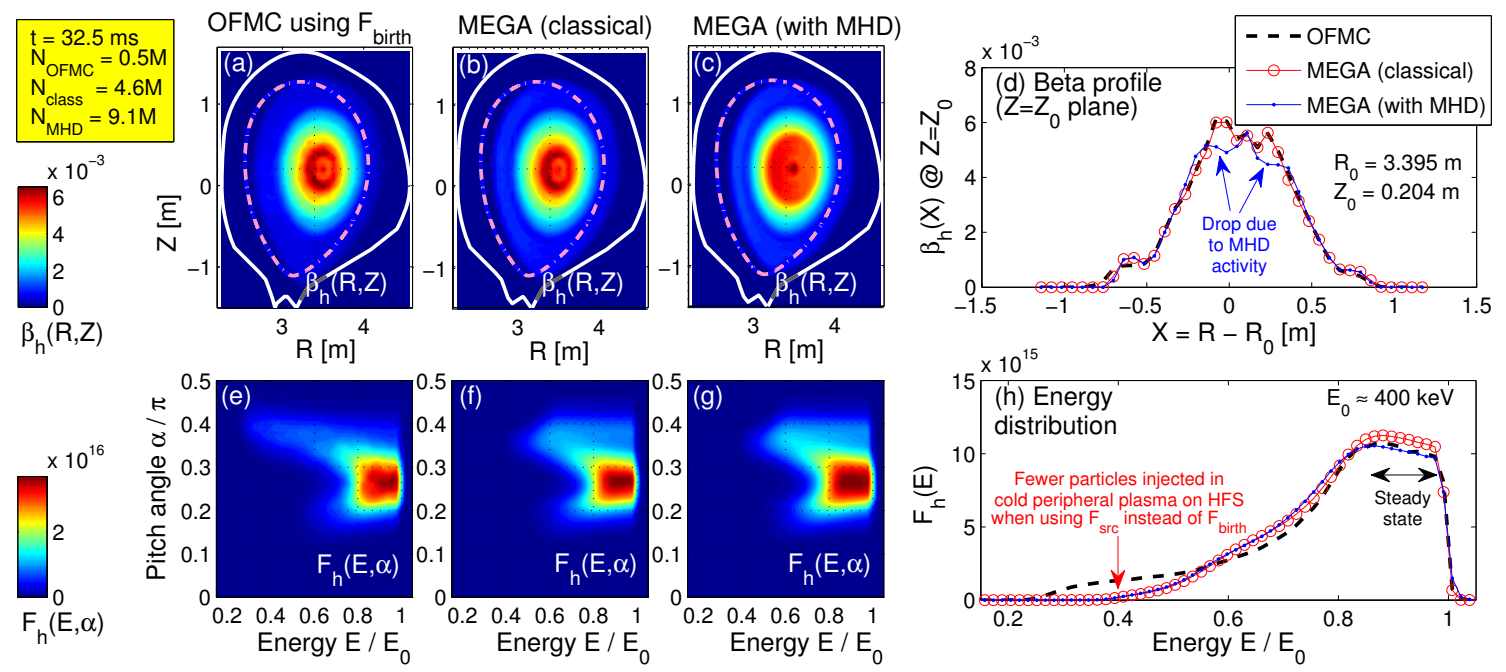

Figure 6. Fast ion distribution at the end of three simulations covering $32.5 \mathrm{~ms}$ after the start of beam injection. The simulations were performed using the OFMC code, the classical MEGA code and the self-consistent MEGA code (with MHD). The respective distributions are represented by $N_{\text {OFMC }}=0.5 \mathrm{M}, N_{\text {class }}=4.6 \mathrm{M}, N_{\mathrm{MHD}}=9.1 \mathrm{M}$ simulation particles. Arranged as Fig. 4.

\subsection{Collisional slow-down and transport of fast ions}

Figure 6 summarizes results of three different simulations covering $32.5 \mathrm{~ms}$ after the start of beam injection. The first was performed with the OFMC code, the second with the classical MEGA code, and the third with the self-consistent MEGA code.

The contour plots of the beta fields in the poloidal plane, $\beta_{\mathrm{h}}(R, Z)$ in Fig. 6(a) and (b), and the corresponding major radial profiles $\beta_{\mathrm{h}}(X)$ evaluated at $Z_{0}=0.204$ $\mathrm{m}$ in Fig. 6(d), show excellent agreement between the OFMC and classical MEGA simulations. Across most of the plasma cross-section, they agree to within 5\%. Only near the plasma boundary - namely, around $X_{\mathrm{hfs}} \approx-0.6 \mathrm{~m}$ on the high-field side and $X_{\mathrm{lfs}} \approx 0.8 \mathrm{~m}$ on the low-field side - the relative difference reaches $30-40 \%$. This discrepancy in the plasma periphery is to be expected, because the equilibrated source distribution $F_{\text {src }}$ used in MEGA does not include particles that are born on prompt-loss orbits, which intersect the wall due to large magnetic drifts. The underlying assumption that collisions are negligible on the short time scale of the first poloidal transit does not hold in the cold and highly collisional plasma near the boundary. There, the rapid collisional slow-down allows these particles to reduce their magnetic drift, so that some of them may remain confined.

A comparison between the velocity distributions computed with OFMC and MEGA confirm this explanation. In Fig. 6(h), one can see that the OFMC simulation contains a group of particles that have already slowed down to $E / E_{0} \approx 0.25(100 \mathrm{keV})$, while all particles in the classical MEGA simulation still have higher energies $E / E_{0} \gtrsim 0.4$ $(160 \mathrm{keV})$. Furthermore, the main differences in Figs. 6(e)-(g) can be seen at large pitch angles $\alpha=\sin ^{-1}\left(v_{\|} / v\right) \approx 0.4 \pi$. This region is populated by particles that are born near 
the high-field side plasma boundary, where the beam lines are almost tangential to $\boldsymbol{B}$.

At $t=32.5 \mathrm{~ms}$, the fast ion distribution has reached a steady state in the energy range $E / E_{0} \gtrsim 0.85(340 \mathrm{keV})$ as indicated in Fig. 6(h). Here, the sources and sinks are already in balance. At lower energies, the ion population still grows as the sources dominate.\|

In the presence of this partially developed high-energy tail in the deuteron distribution, resonantly driven modes with low toroidal mode numbers $n \gtrsim 1$ are already destabilized (cf. Fig. 4 of Ref. [18]). Figure 6(d) shows that, as a result of this low- $n$ MHD activity, the radial profile of the fast ion pressure in the core plasma near $X \approx 0$ is reduced by as much as $20 \%$. The properties and dynamics of these MHD modes are analyzed in the following Sections 3.2-3.4.

Note that, in the simulations discussed in this work, we do not observe any significant influence of the relatively steep velocity space gradients seen in Fig. 6. The dominant modes in the present simulations are primarily driven by radial gradients of the fast ion pressure, which is not unusual for modes with high Alfvénic frequencies $\left(\omega \sim \omega_{\mathrm{A}}\right)$ and low but nonzero toroidal mode numbers $(n \gtrsim 1)$. $\mathcal{P}$ Meanwhile, indirect effects of velocity space gradients - in particular, via zonal structures and other $n=0$ modes that they can drive - are absent in the present simulations, because we substitute into Eq. (4) only the $n=1,2,3$ harmonics of $\boldsymbol{j}_{\mathrm{h} \text {,eff }}$. Thus, the $n=0$ harmonic is driven only via MHD nonlinearities here, and we will ignore it in the following because its amplitude turns out to be typically 2 orders of magnitude smaller than the dominant $n=1$ harmonic.

\subsection{Physical interpretation of chirping modes as bursts of $n=1$ EPMs}

Figure 7(a) shows a few bursts of chirping modes that occurred during the time window $4340 \mathrm{~ms} \leq t \leq 4375 \mathrm{~ms}$ after an ALE in shot E048424. Clearly, the $n=1$ signal dominates during the chirping modes in the experiment. Figure 7(b) shows that similar bursty mode activity dominated by $n=1$ is reproduced in the self-consistent MEGA simulations. Note that, due to the non-slip boundary condition $(\delta \boldsymbol{u}=0)$ used in the simulations, we are not able to measure magnetic fluctuations at the wall as in the experiments. Instead, we have to use quantities measured in the plasma interior. This caveat limits somewhat the comparability of experimental and simulation results. The "amplitude" $A_{n}(t)=W_{n}^{1 / 2}(t)$ plotted in Fig. $7(\mathrm{~b})$ is the square root of the volumeintegrated fluctuation energy $W_{n}=\frac{1}{2} \int \mathrm{d}^{3} x\left(\left|\rho_{\mathrm{b}}^{1 / 2} \delta \boldsymbol{u}\right|_{n}^{2}+|\delta \boldsymbol{B}|_{n}^{2}\right)$ for $n>0$.

In order to clarify the physical nature of the chirping modes, it is essential to determine the spatial structure of the fluctuations in addition to measuring their frequency spectrum. However, in the JT-60U experiments, it was not possible to obtain

\|In the present case, it takes about $500 \mathrm{~ms}$ for the classical fast ion tail to reach a steady state down to thermal energies of a few $\mathrm{keV}$.

$\mathcal{P}$ This can be anticipated from the conservation of the quantity $C=\omega_{n} P_{\zeta}-n E$, which would hold for the time-dependent canonical toroidal momentum $P_{\zeta}(t)$ and kinetic energy $E(t)$ of a particle subject to a fluctuating field characterized by a single toroidal harmonic $n$ and constant frequency $\omega_{n}$. 


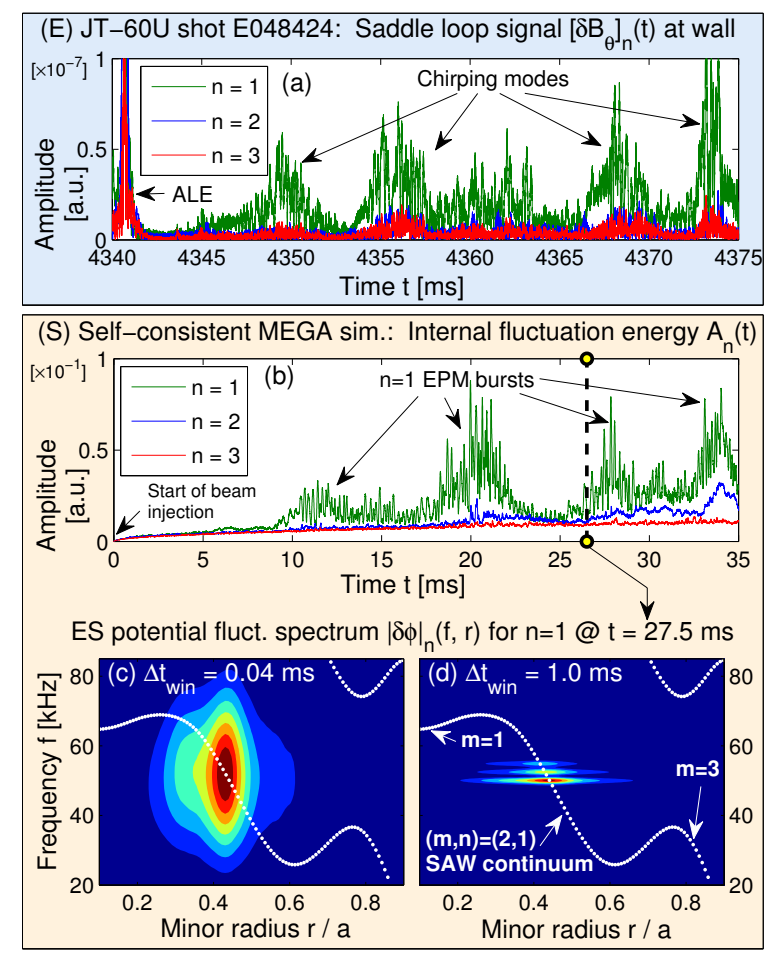

Figure 7. The chirping modes observed in JT-60U experiments (E) are reproduced as bursts of $n=1$ energetic particle modes (EPM) in the self-consistent MEGA simulation (S). Panels (a) and (b) show the time traces of mode amplitude for $n=1,2,3$. For the dominant $n=1$ harmonic in the simulation, panels (c) and (d) show a snapshot of the electrostatic (ES) potential fluctuation spectrum $|\delta \phi|_{n=1}(f, r)$ in the frequency-radius plane obtained with FFTs over two different time windows: (c) $\Delta t_{\text {win }}=0.04 \mathrm{~ms}$ and (d) $1 \mathrm{~ms}$. White dotted lines represent the shear Alfvén wave (SAW) continua in the slow-sound approximation with poloidal mode numbers $m=1,2,3$.

information about the radial location of the modes. Soft X-ray diagnostics and detectors used for reflectometry and interferometry were unavailable due to the high neutron fluxes, and the electron cyclotron emission (ECE) diagnostics could not be operated at the low magnetic field strength around $B \approx 1 \mathrm{~T}$. Thus, for the radial mode structure, we will have to rely entirely on the results of our "numerical experiment".

Assuming that the MHD velocity is dominated by $\boldsymbol{E} \times \boldsymbol{B}$ drift, we compute the electrostatic potential $\delta \Phi(r, \vartheta, \zeta, t)=\sum_{n} e^{-i n \zeta} \delta \phi_{n}(r, \vartheta, t)$ from $\delta \boldsymbol{u} \approx \frac{1}{B} \hat{\boldsymbol{b}} \times \nabla \delta \Phi$. Here, $r$ is the volume-averaged minor radius of the plasma, with $0 \leq r(\psi) / a \leq 1$. Next, using a fast Fourier transform (FFT) weighted by a Hanning time window $H(\tau-t)$ of size $\Delta t_{\text {win }}$, we compute the fluctuation spectrum $|\delta \phi|_{n}(\omega, r)$ in the frequency-radius plane as

$$
|\delta \phi|_{n}\left(\omega, r \mid \vartheta_{0}, t\right)=\left|\int \mathrm{d} \tau \delta \phi_{n}\left(r, \vartheta_{0}, \tau\right) H(\tau-t) e^{i \omega(\tau-t)}\right| .
$$

Here, we use the field values at poloidal angle $\vartheta_{0}=0$; i.e., on the low-field side of the magnetic axis. The Hanning window is padded with zeros $\left(\Delta t_{\text {pad }}=8 \times \Delta t_{\text {win }}\right)$ on both sides in order to sharpen the image. The frequency will be given as $f=\omega /(2 \pi)$ in $\mathrm{kHz}$.

For a snapshot taken at $t=27.5 \mathrm{~ms}$, Fig. $7(\mathrm{c})$ and (d) shows the fluctuation spectra 
$|\delta \phi|_{n=1}(f, r)$ for the dominant $n=1$ harmonic and two different Hanning window sizes: $\Delta t_{\text {win }}=0.04 \mathrm{~ms}$ and $1.0 \mathrm{~ms}$. One can see that the $n=1$ signal originates from a resonant destabilization of the shear Alfvén wave (SAW) continuum branch with poloidal mode number $m=2$. This suggests that the chirping modes seen in JT-60U are bursts of $5 n=1$ energetic particle modes (EPM [30]). This result of the self-consistent MEGA simulation confirms the predictions of previous conventional initial-value simulations (beginning with Ref. [31]).

A similar analysis (not shown) was performed for the higher-order harmonics $n=2$ and $n=3$. It is found that the $n=2$ dynamics are primarily the result of distortions of the fundamental $n=1$ mode: they are observed in the same radial domain as the $n=1$ mode and mirror the $n=1$ chirps at twice the frequency. The $n=3$ dynamics consist of fluctuations driven by MHD nonlinearities as well as a fast-ion-driven $n=3$ mode similar to that described in Ref. [18]. Note that the results reported in Ref. [18] were obtained for the zero-Larmor-radius limit, whereas the present simulations include the effect of the large fast ion Larmor radii via gyroaveraging. This reduces the efficiency of resonant interactions with increasing toroidal mode number $n$. For instance, in the case of $n=3$, gyroaveraging was shown to increase the threshold for the transition from weak to strong mode activity [17]. This is one of the main reasons why the $n=3$ mode amplitude remains relatively low during the initial $35 \mathrm{~ms}$ interval analyzed here.

\subsection{Frequency chirping on the millisecond scale}

If one sacrifices resolution in time and uses a larger FFT window $\Delta t_{\text {win }}=1.0$ ms as in Fig. $7(\mathrm{~d})$, the fluctuation spectrum $|\delta \phi|_{n=1}(f, r)$ exhibits multiple sharp peaks at different frequencies. The motion of these peaks along the $(m, n)=(2,1)$ SAW continuum branch causes up- and downward frequency chirping observed on the millisecond scale, which is the topic of this section.

Large FFT windows with sizes around $\Delta t_{\text {win }}=1.0 \mathrm{~ms}$ have been routinely used in previous analyses of experimental data $[1,2,3,4]$, producing spectrograms like those in Figs. 1 and 2. The complete data analysis procedure goes as follows. Starting from the raw Mirnov coil signals, which are sampled at $0.5-1 \mathrm{MHz}$ and are proportional to $\delta \dot{B}_{\vartheta}$, the magnetic fluctuation signal is recovered via cumulative trapezoidal integration: $\delta B_{\vartheta}(t)=\int_{0}^{t} \mathrm{~d} t^{\prime} \delta \dot{B}_{\vartheta}\left(t^{\prime}\right)$. Then, a $30-80 \mathrm{kHz}$ band-pass filter is applied, using zero-phase forward and reverse digital filtering with a 2nd-order Butterworth filter.

Figure $8(\mathrm{a})$ shows the magnetic fluctuation signal $\delta B_{\vartheta}(t)$ for the time window $3930 \mathrm{~ms} \leq t \leq 3965 \mathrm{~ms}$ of JT-60U shot E036378, immediately after the revival of the $\mathrm{N}-\mathrm{NB}$ system. Figure $8(\mathrm{c})$ shows $\delta B_{\vartheta}(t)$ for the time window $4340 \mathrm{~ms} \leq t \leq 4375 \mathrm{~ms}$ of shot E048424, immediately after an ALE, whose large amplitude $\left( \pm 40 \times 10^{-5}\right)$ is not fully shown. FFTs over a Hanning time window of size $\Delta t_{\text {win }}=1.0 \mathrm{~ms}$ advancing at time steps of size $\Delta t_{\text {step }}=0.2 \mathrm{~ms}$ yield the spectrograms shown in Figs. 8(b) and (d).

The self-consistent MEGA simulation directly yields the magnetic fluctuation signal $\delta B_{\vartheta}(t)$ anywhere inside the plasma boundary. Figure 8(e) shows the evolution of the 

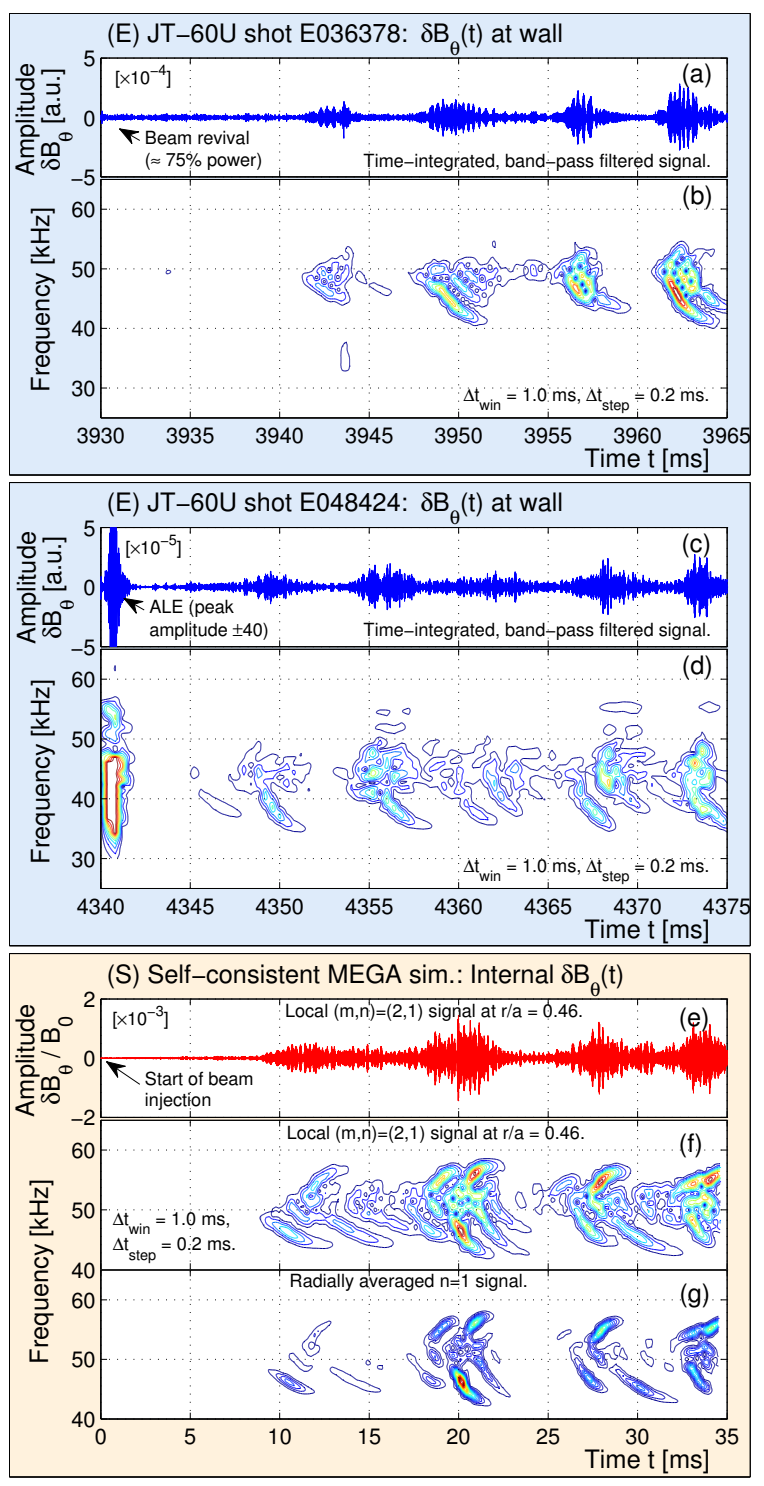

Figure 8. Comparison between magnetic fluctuation signals and the resulting spectrograms in experiments (E) and simulation (S). The spectrograms are plotted using a linear color scale and were computed using FFTs over a running time window with large size $\Delta t_{\text {win }}=1 \mathrm{~ms}$.

cosine component of its $(m, n)=(2,1)$ harmonic evaluated at radius $r / a=0.46$ and sampled at $288 \mathrm{kHz}$. One can see in Fig. 8(e) that the $n=1$ EPMs occur in bursts that last a few ms each and are separated by intervals of about 5-10 ms. The corresponding spectrogram in Fig. 8(f) shows alternating down- and upward chirps that last about 1-5 ms and extend about $\pm(5-10) \mathrm{kHz}$ around $50 \mathrm{kHz}$. Note that the detailed form of the chirps seen in the spectrogram may vary somewhat depending on the radial location and the poloidal harmonic for which it is evaluated. In order to illustrate this, Fig. 8(g) shows another spectrogram obtained from the radially averaged $n=1$ signal, summed over all poloidal harmonics. ${ }^{+}$

+ MEGA solves the MHD equations directly in cylinder coordinates $(R, Z, \varphi)$. The results are then 


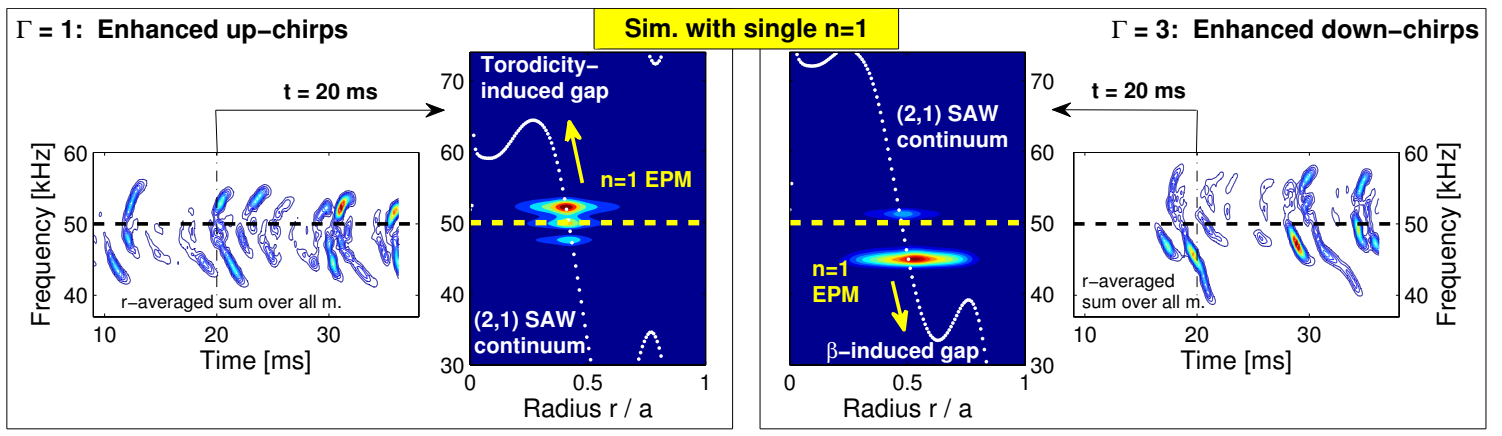

Figure 9. Comparison between chirping behavior of the $n=1 \mathrm{EPM}$ in self-consistent MEGA simulations run with different values of the specific heat ratio: $\Gamma=1$ (left) and $\Gamma=3$ (right). Here, only fluctuations with toroidal mode number $n=1$ were simulated and the size of the Hanning window used for the FFTs is $\Delta t_{\text {win }}=1.0 \mathrm{~ms}$. The two panels in the center show snapshots of the fluctuation spectra of the ES potential taken at $t=20 \mathrm{~ms}$ in the respective simulation. Arrows indicate the direction into which the spectral peak moves along the $(m, n)=(2,1)$ SAW continuum at that time.

These simulation results (burst duration, burst intervals, frequency range) are qualitatively and quantitatively similar to the experimental results in many shots, including E036378 and E048424 shown in Fig. 8(a)-(d). In these particular cases, one can even see a comparable delay of about $5-10 \mathrm{~ms}$ before the first low-amplitude bursts of chirping modes become visible after the (re)start of N-NB injection (E036378 and simulation) or after the ALE (E048424).

However, one can also see some differences in the details; not only between simulation and experiment, but also among the experimental results. One quantitative difference worth noting is that the chirping range may be shifted up or down by about 5-10 kHz. As was discussed in Section 1, this can be attributed to different densities and beta values in different cases. On the qualitative side, one can see variations in the dominant direction of chirping, which may also depend on the plasma parameters. Indeed, in Section 2 of Ref. [32], we have argued that the structure of the SAW continuum may play an important role in determining the chirping direction of an EPM. This means that the various plasma parameters do not necessarily change the chirping behavior directly, but they may do so by altering the location of the continuous spectra relative to the fast ion resonances.

In order to demonstrate this, we employ a convenient method used in our recent sensitivity study [17], where we varied the ratio of specific heats $\Gamma$ between the extreme limits $\Gamma=1$ and $\Gamma=3$. The results of self-consistent MEGA simulations of an $n=1$ EPM for $\Gamma=1$ and 3 are summarized in Fig. 9. In both cases, the chirps start near the same frequency of $50 \mathrm{kHz}$. This is because the geometry of the MHD equilibrium and the fast ion orbits are identical, so the resonances remain fixed as well. The specific mapped to toroidal flux coordinates $\left(\psi, \vartheta_{\mathrm{f}}, \zeta\right)$, decomposed into poloidal and toroidal Fourier harmonics $(m, n)$ and stored for further processing. In the present simulations, harmonics with $0 \leq m \leq 12$ and $1 \leq n \leq 4$ are recorded, each with 101 radial grid points uniformly spaced in $0 \leq \psi \leq 1$. 
heat ratio controls plasma compressibility in the equation of state (8), so it influences the size of the compressibility-induced (or " $\beta$-induced") low-frequency gap.

When $\Gamma$ is reduced to 1 (Fig. 9, left), the SAW continua are shifted downward, so that the $n=1 \mathrm{EPM}$ is excited closer to the accumulation point of the toroidicity-induced

5 gap. Here, it appears that the amplitudes of the up-chirping signals are somewhat enhanced. When $\Gamma$ is increased to 3 (Fig. 9, right), the SAW continua are shifted upward, so that the $n=1 \mathrm{EPM}$ is excited closer to the accumulation point of the $\beta$-induced gap. Here, it appears that the down-chirping signals are enhanced. In both cases, chirps in the respective other direction prevail, so the effect is not large. This parameter $\Gamma$.

Since the mode dynamics are very complex (multiple interfering short-lived chirping modes) and since the fast ion dynamics are still under investigation, we are not yet able to offer a definitive explanation for why the $\Gamma$-induced changes in the SAW continua alter the chirping behavior in the particular way seen in Fig. 9. The reduced phase mixing ("continuum damping") that the wave packets experience closer to an accumulation point is likely to play a role. However, other factors (such as resonance conditions, the associated power transfer rates, and nonlinear phase-space structures) would also have to be considered.

\subsection{Sub-millisecond pulsations and phase jumps due to EPM beating}

Spectrograms computed with low temporal resolution on the millisecond time scale, such as those in Figs. 8 and 9, give the impression that there are modes that smoothly chirp upward or downward with slowly varying amplitudes. However, it has been previously demonstrated in other experiments $[14,15]$ that the mode amplitudes are subject to rapid pulsations on the sub-millisecond time scale and that there are often abrupt phase jumps between these pulses. In this section, we revisit these phenomena, using measurements made in JT-60U and in our self-consistent MEGA simulations. On the basis of these results, a simple explanation will be proposed afterwards.

Figures 10 and 11 show in detail what happens from the point of view of the magnetic fluctuation signals during a typical downward chirp. In both figures, the boxes on the left show a $5 \mathrm{~ms}$ time window covering one complete burst of $n=1 \mathrm{EPM}$ activity. The boxes on the right show a further magnified view of a $1 \mathrm{~ms}$ time window, where one can see what happens on the sub-millisecond scale.

The first panel in each box in Figs. 10 and 11 shows the time trace of the local magnetic fluctuation signal $\delta B_{\vartheta}(t)$. As before, the experimental $\delta B_{\vartheta}(t)$ signal is acquired at the wall. ${ }^{*}$ For the simulation, we show the evolution of the $(m, n)=(2,1)$ harmonic

* Since the magnetic fluctuations in the experiments are measured at the wall, one may expect them to be polluted by other signals, such as modes with $n \neq 1$ and fluctuations near the plasma boundary. However, the good agreement that we find between experiment and simulations suggest that the internal chirping modes with $n=1$ dominate the experimental signal in the $30-70 \mathrm{kHz}$ band by far. 


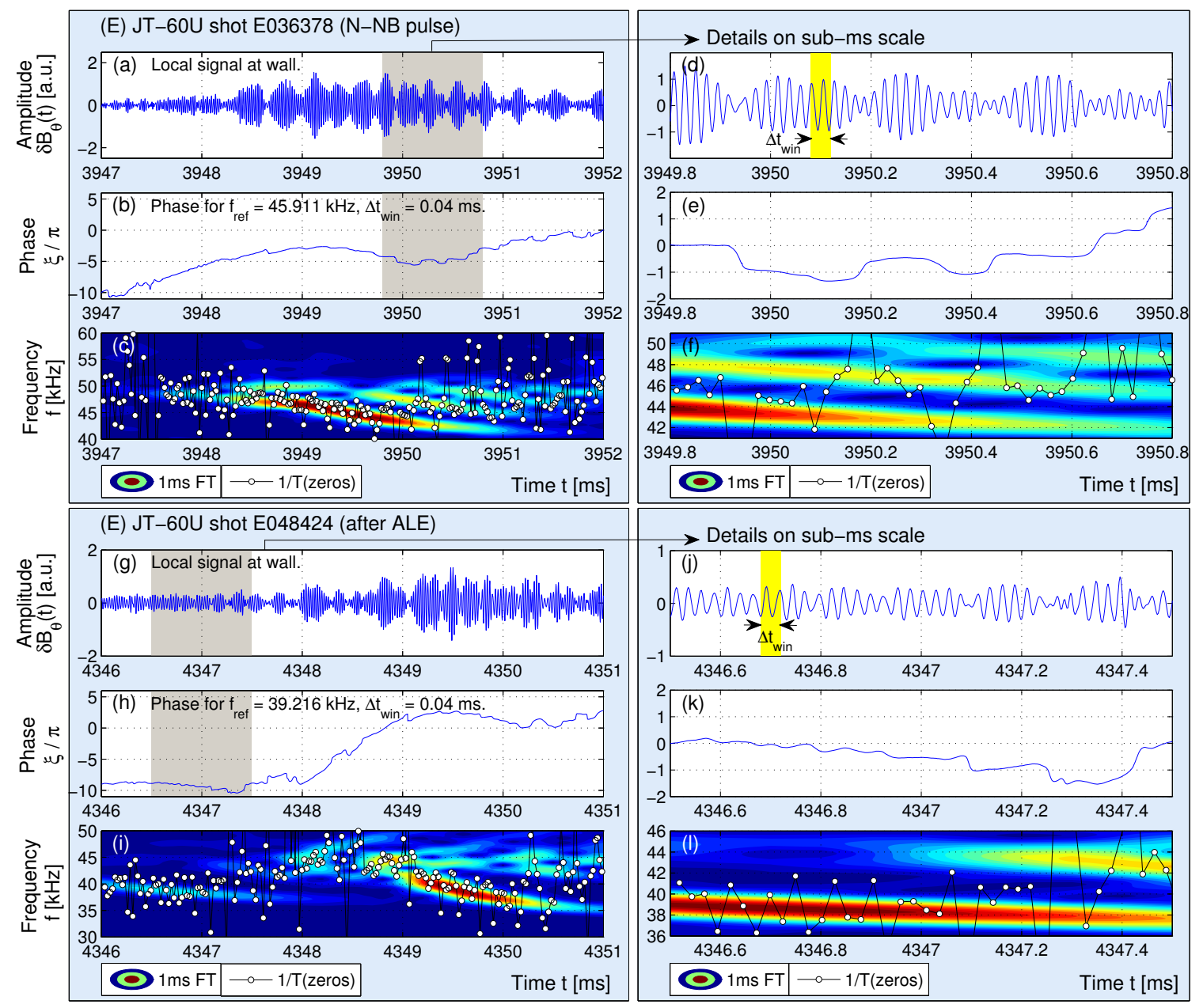

Figure 10. Pulsations, phase jumps and frequency chirping in the magnetic fluctuation signals from JT-60U shots E036378 (top) and E048424 (bottom). The boxes on the left show a $5 \mathrm{~ms}$ interval covering one burst of mode activity. The sub-millisecond details can be seen in the boxes on the right, where a $1 \mathrm{~ms}$ interval is enlarged. Each box shows the time traces of the magnetic fluctuation amplitude $\delta B_{\vartheta}(t)$, the instantaneous complex phase $\xi(t)$ defined in Eq. (30), and the wave frequency $f(t)$ measured with low temporal resolution (1 ms FT spectrograms, color contours) and high resolution (inverse of the oscillation period $T(t) \sim 0.2-0.25 \mathrm{~ms}$, circles).

of $\delta B_{\vartheta}$ evaluated at $r / a=0.46$. One can clearly see the wave oscillations with periods in the range $T=1 / f \approx 0.020-0.025 \mathrm{~ms}(40-50 \mathrm{kHz})$. Moreover, the waves occur in pulses that last about $0.2-0.3 \mathrm{~ms}$ each. If one looks closely, one can see phase jumps between many of the pulses.

In order to visualize the phase jumps more clearly, we measure the instantaneous complex wave phase $\xi(t)$ of the signal relative to oscillations with a given reference frequency $f_{\text {ref }}=\omega_{\text {ref }} /(2 \pi)$ :

$$
\xi(t)=\arg \left\{\int \mathrm{d} \tau \delta B_{\vartheta}(\tau) H(t-\tau) e^{-i \omega_{\text {ref }}(t-\tau)}\right\} .
$$

The time traces of $\xi(t)$ are plotted in the second panel of each box in Figs. 10 and 11. 

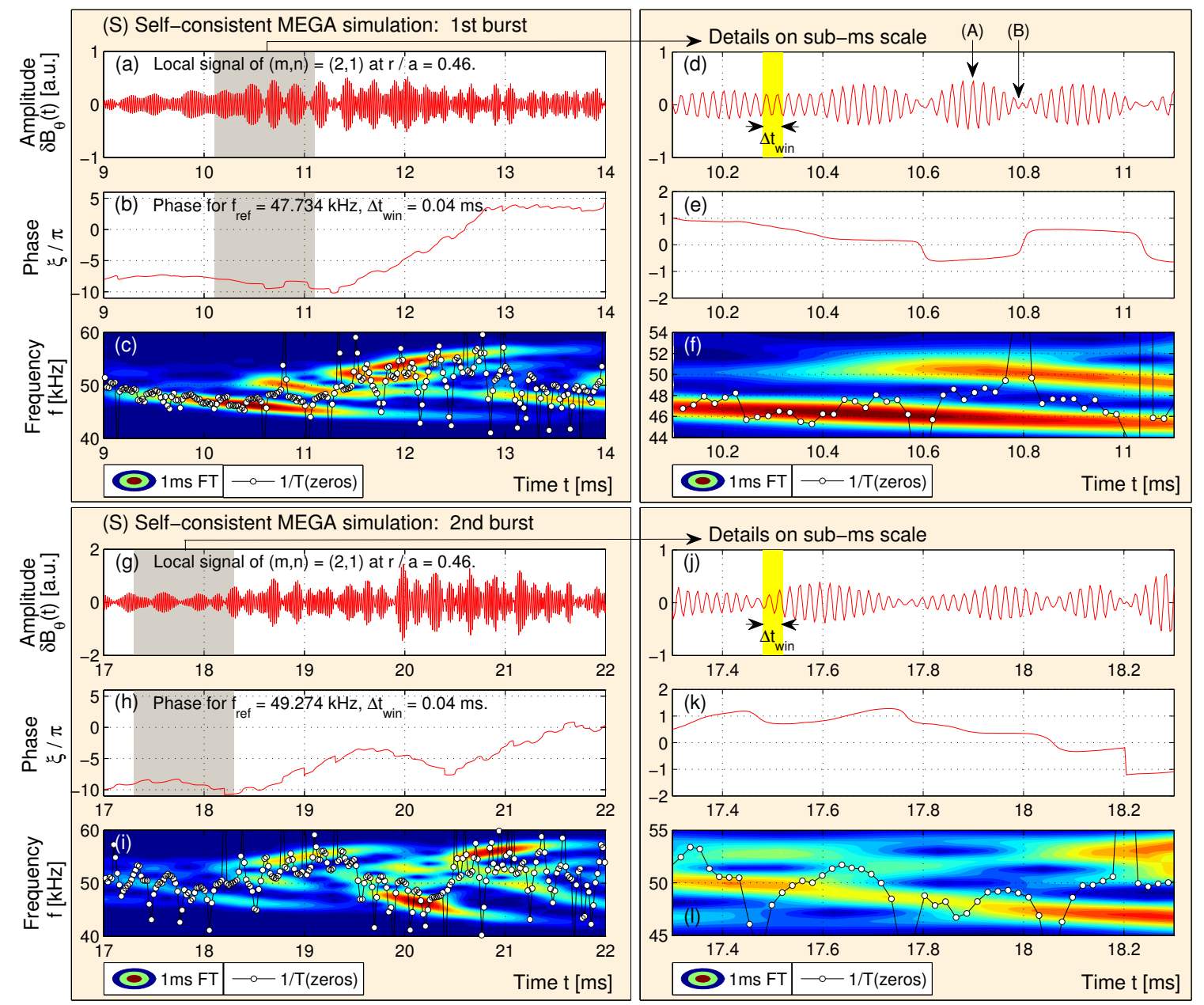

Figure 11. Pulsations, phase jumps and frequency chirping in the magnetic fluctuation signals during the first (top) and second burst (bottom) of $n=1 \mathrm{EPM}$ activity in the self-consistent MEGA simulation. Arranged as Fig. 10. In panel (d), the labels (A) and (B) indicate the times at which the snapshots shown in Fig. 12 were taken.

For the experimental data in Fig. 10 we have chosen $f_{\text {ref }}=45.911 \mathrm{kHz}$ for E036378 and $39.216 \mathrm{kHz}$ for E048424, respectively. For the simulation data in Figs. 11 we have chosen $f_{\text {ref }}=47.734 \mathrm{kHz}$ for the first burst and $49.274 \mathrm{kHz}$ for the second burst. In order to maximize the temporal resolution, the Hanning window $H(t)$ in Eq. (29) was 5 chosen to have a small width of only $\Delta t_{\text {win }}=0.04 \mathrm{~ms}$. Basically, Eq. (29) means that we are convolving the magnetic signal $\delta B_{\vartheta}(t)$ with a wavelet-like basis function $H(\tau) e^{-i \omega_{\text {ref }} \tau}$ whose width barely encompasses two typical wave oscillation periods.

One can see in Figs. 10 and 11 that, both in experiment and simulation, the phase $\xi(t)$ changes most rapidly between successive instability pulses; i.e., when the wave amplitude is small. In particular, when the wave amplitude drops to nearly zero, the phase performs jumps of size $\Delta \xi= \pm \pi$. Similar pulsations and phase jumps were previously reported in JET and HL-2A tokamak experiments [14, 15], and they are reproduced here in self-consistent numerical simulations for the first time. 


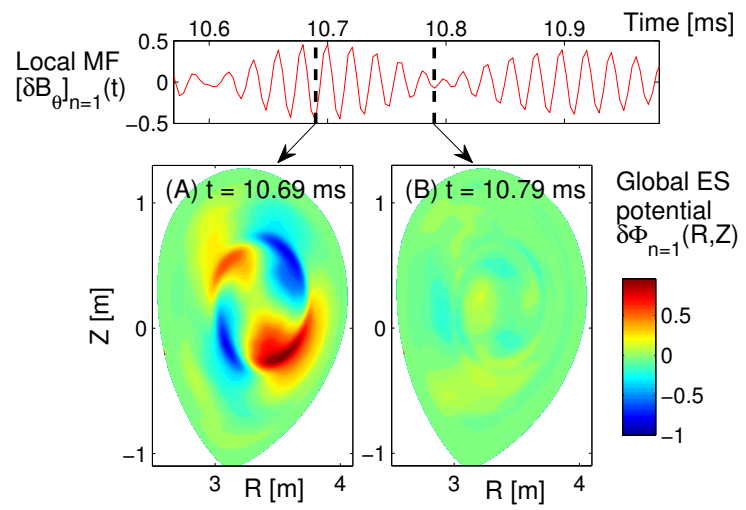

Figure 12. Snapshots of the global structure of the electrostatic (ES) potential in the simulation shown in Fig. 11. One can clearly see the global character of the constructive and destructive interference at the peak of a pulse (A) and between two pulses (B), respectively.

Although the time traces of $\delta B_{\vartheta}(t)$ shown in the top panels of Figs. 10 and 11 were measured at only one point in space, the pulsations they show are not localized. As can be seen in Fig. 12, the entire global mode disappears between successive pulses.

The bottom panel of each box in Figs. 10 and 11 contains a contour plot of the 5 spectrogram computed from the local $\delta B_{\vartheta}(t)$ signal using a large Hanning window size $\Delta t_{\text {win }}=1 \mathrm{~ms}$. The chirps seen here amount to only $10-20 \%$ of the mode frequency, so they are difficult to detect by eye in the time traces of the magnetic fluctuation $\delta B_{\vartheta}(t)$. One way of obtaining detailed information about the evolution of the wave frequency while also retaining high temporal resolution is to plot the time trace of the inverse wave period $1 / T$. There are several ways to measure $T$, and they all give very similar results. Overlaid on the spectrograms in the bottom panel in each box of Figs. 10 and 11, we show the evolution of $1 / T$ measured between successive zero-crossings from $\delta B_{\vartheta}(t)>0$ to $\delta B_{\vartheta}(t+\Delta t)<0$.

As they must by definition, the overall trends of the $1 / T(t)$ curves agree with the gradual down- or upward chirping of the oscillation frequency seen on the $1 \mathrm{~ms}$ time scale. However, one can also observe in Figs. 10 and 11 that, on the sub-millisecond time scale, the instantaneous frequency $1 / T(t)$ deviates substantially from the spectrograms computed with low temporal resolution. Such discrepancies are to be expected since high resolution in time and high resolution in frequency cannot be achieved simultaneously. Each quantity plotted in Figs. 10 and 11 captures only a certain aspect of the signal while distorting others, and the "truth" may be thought to lie somewhere inbetween.

\section{Discussion of chirping and beating}

The observations described in the previous section 3.4 give rise to the following questions: What is the reason for the pulsations and phase jumps? And how much of the chirping that was seen on the millisecond time scale is "true chirping" in the sense that the 


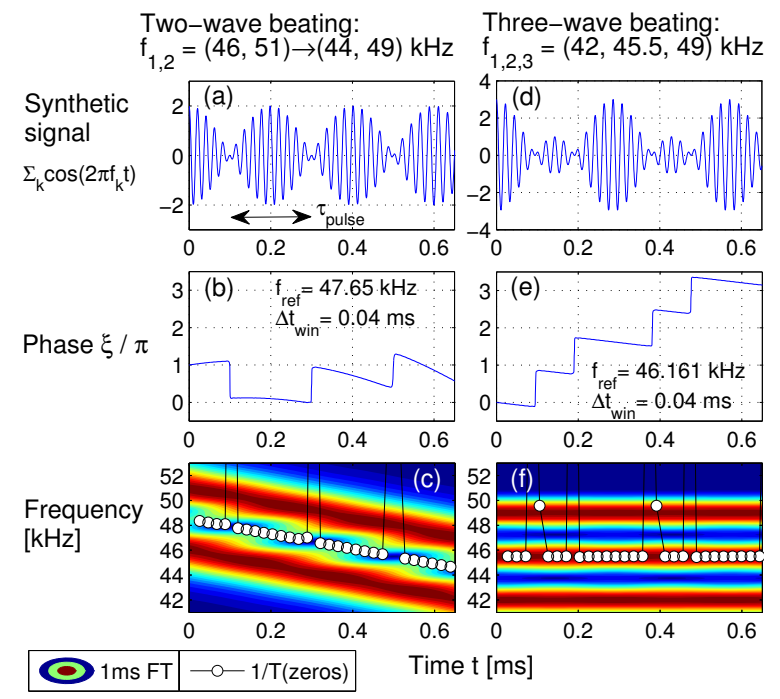

Figure 13. Analysis of synthetic signals for two-wave (left) and three-wave beating (right), showing pulsations, phase jumps and the equality between the inverse oscillation period and the mean frequency, $1 / T(t)=\bar{f}(t)$.

oscillation frequency of a given mode or wave packet gradually increases or decreases? The following discussion of these questions is intended to serve as a starting point and motivation for more detailed studies of the underlying wave-particle interactions.

Many features seen in the time traces of $\delta B_{\vartheta}(t), \xi(t)$ and $1 / T(t)$ in Figs. 10 and 11 are reminiscent of beat waves. For illustration and comparison, Fig. 13 shows results of the same analysis applied to synthetic signals that are composed of multiple cosine functions $\sum_{k} A_{k} \cos \left(2 \pi f_{k} t\right)$. For simplicity, we let $A_{k}=1$ for all $k=1,2, \ldots$

Panels (a)-(c) on the left-hand side of Fig. 13 show an example with two superimposed oscillations with different downward-chirping frequencies: $f_{1}=46 \rightarrow 42$ $\mathrm{kHz}$ and $f_{2}=51 \rightarrow 47 \mathrm{kHz}$. This case has been set up to be similar to the situation in Fig. 11(d)-(f) during the time window $10.6 \mathrm{~ms} \lesssim t \lesssim 11.1 \mathrm{~ms}$. The pulse length $\tau_{\text {pulse }}=1 /\left(f_{2}-f_{1}\right)=0.2 \mathrm{~ms}$, the phase jumps $\Delta \xi= \pm \pi$ and the inverse period $1 / T=\left(f_{1}+f_{2}\right) / 2=48.5 \rightarrow 44.5 \mathrm{kHz}$ are clearly reproduced.

Panels (d)-(f) on the right-hand side of Fig. 13 show an example with three superimposed oscillations with equidistant constant frequencies $f_{1}=42 \mathrm{kHz}, f_{2}=45.5$ $\mathrm{kHz}$ and $f_{3}=49 \mathrm{kHz}$. Except for ignoring the evolution of the frequencies $f_{k}$, this case is similar to the situation in Fig. 10(d)-(f) during the time window $3950.4 \mathrm{~ms} \lesssim$ $t \lesssim 3950.8 \mathrm{~ms}$. The alternating small- and large-amplitude pulses with pulse lengths $\tau_{\text {pulse }}^{(1)}=1 /\left(f_{3}-f_{1}\right)=0.2 \mathrm{~ms}$ and $\tau_{\text {pulse }}^{(2)}=\tau_{\text {pulse }}^{(1)} / 3=0.067 \mathrm{~ms}$, the phase jumps $\Delta \xi=+\pi$ and the inverse period $1 / T=\left(f_{1}+f_{2}+f_{3}\right) / 3=45.5 \mathrm{kHz}$ are clearly reproduced.

These results suggest that the pulsations and phase jumps are indeed a consequence of the beating between multiple coexisting $n=1$ EPMs.

From the observation of perfect destructive interference that makes the mode amplitude vanish between the beats, one can infer that the interfering waves have similar amplitudes around those times. Thus, the beating phenomenon can be used 
to obtain additional information about the evolution of the individual amplitudes of the superimposed waves on the sub-millisecond time scale, where the low-resolution spectrograms are only of little use because the amplitudes are smeared over $\pm 0.5 \mathrm{~ms}$.

Besides all the agreement between the synthetic beats and those seen in simulations

it is clear that a linear time-dependence of the frequencies $f_{k}(t) \propto t$ implies $\xi(t) \propto t^{2}$. Indeed, such a parabolic time-dependence can be clearly seen in Fig. 13(b) if one subtracts out the phase jumps. However, one is hard pressed when trying to recognize a similar parabolic evolution of the phase $\xi(t)$ in Figs. 10(e) and 11(e). Instead, $(\xi(t)=$ const. $)$ or vary linearly with time $(\xi(t) \propto t)$ for the duration of each pulse. By virtue of Eq. (30), this would imply that the mean oscillation frequency $\bar{f}=N^{-1} \sum_{k=1}^{N} f_{k}$ either matches the reference frequency $f_{\text {ref }}$, or that there is a constant frequency mismatch, $\bar{f}-f_{\text {ref }}=$ const. is difficult to draw strong conclusions. But if our observation described in the preceding paragraph is correct, it means that the low-resolution spectrograms such as those in Figs. 11(l) and 11(f) may be misleading in the sense that the frequency chirping they show reflects only the trends on the millisecond time scale. On the sub-millisecond pulse and vary in discrete steps from one pulse to the next. The frequency may even temporarily chirp in the opposite direction. Such details may be important for the development of theories for chirping modes driven by fast ions.

As a counter-example, Fig. 14 shows the results of an analysis of shot E039672, is not present in the $q(\rho)$ profile used in our simulations.

It is possible that the particles trapped around the resonance are able to perform many nonlinear bounces during the relatively long time scale $\tau_{\text {pulse }} \sim 5$ ms of the mode pulse seen in Fig. 14 for $t \gtrsim 4396$. If so, the dynamics of such a long-lived mode may be captured by theories that assume a large separation between the time scales for wave where a burst of beating modes is followed by a long-lived mode that performs smooth upward frequency chirping without phase jumps or rapid pulsations. Unfortunately, we are not able to classify this mode, since it was not reproduced in our simulations, so we do not know its radial structure. Our guess is that it is a core-localized shear Alfvén mode chirping along continuous spectra in a region of negative magnetic shear, which pulsation and particle bouncing: $\tau_{\text {pulse }} \gg \tau_{\text {bounce. }}$ In contrast, the short pulses (beats) 


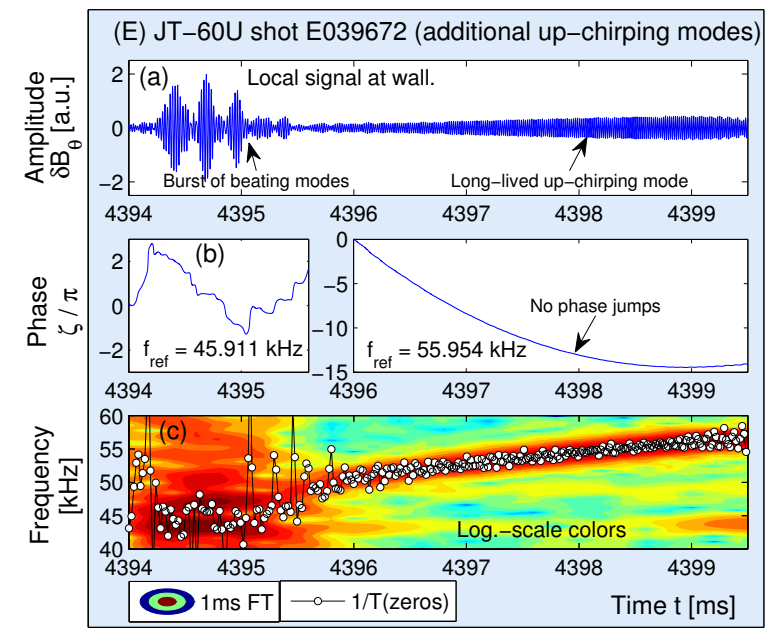

Figure 14. Analysis of shot E039672 showing how the evolution of the phase $\xi(t)$ and frequency $1 / T(t)$ differs between a burst of beating modes ( $4394 \mathrm{~ms} \lesssim t \lesssim 4395.5 \mathrm{~ms}$ ) and the subsequent long-lived mode that performs true upward chirping. Note that, at other times, these two kinds of modes appear simultaneously or in reversed order, which suggests that they are independent and have different spatial structures.

that dominate in our simulations and in many JT-60U experiments and whose periods are around $\tau_{\text {pulse }} \sim 0.2 \mathrm{~ms} \sim 10 \times \tau_{\text {wave }}$, suggest that the two time scales are comparable: $\tau_{\text {pulse }} \sim \tau_{\text {bounce. }}$. In fact, there may be a close physical link between the beating and wave-particle trapping.

These results and interpretations motivate a detailed analysis of the nonlinear interactions between fast ions and rapidly pulsating MHD waves in future work. In order to further emphasize the importance of sub-millisecond dynamics, let us note that the typical life time of an ALE is also about $0.3 \mathrm{~ms}$; i.e., similar to the beating period of the $n=1$ EPMs studied above. The main differences are that ALEs have 4-10 times larger fluctuation amplitudes $\delta B_{\vartheta}$ and a larger harmonic content $(n=1,2,3)$ [33].

\section{Conclusion}

In this paper, we have demonstrated that the extended hybrid code MEGA is capable of reproducing multiple bursts of chirping Alfvén modes that were routinely observed in N-NB-driven JT-60U tokamak experiments and originally dubbed "fast frequency sweeping (fast FS) modes".

The extended simulation model was described in detail. It consists of full MHD equations, fast ion guiding center equations of motion with FLR effects, and was now extended with realistic fast ion sources and collisions, so that it self-consistently captures dynamics across a wide range of time scales $(0.01-100 \mathrm{~ms})$. The model for fast ion sources and collisions is similar to that used in the well-established orbit-following Monte-Carlo code OFMC [22], and MEGA was successfully benchmarked against OFMC.

It is demonstrated that the experimentally observed chirping phenomena can be 
attributed to bursts of energetic particle modes (EPM [34]) with dominant toroidal mode number $n=1$. This result of the self-consistent MEGA simulation confirms the predictions of previous conventional initial-value simulations that were initialized with an analytical slowing-down model distribution $[10,13,31,35,36]$ or with a classical fast 5 ion distribution computed by the OFMC code $[18,37]$.

On the long time scale of 1-10 ms, the self-consistent MEGA simulation of chirping modes reproduces the chirping range $(40-60 \mathrm{kHz})$, duration (few ms) and intervals (5-10 $\mathrm{ms}$ ) of the bursts. By varying the plasma compressibility via the specific heat ratio $\Gamma$ in the equation of state (8), we demonstrated that the structure of the shear Alfvén experimentally measured plasma profiles used for the equilibrium reconstruction, this may explain some of the differences seen between chirping mode dynamics in different experiments and our simulation (e.g., dominant up- or downward chirping).

On the short time scale of $0.01-0.1 \mathrm{~ms}$, the simulation reproduces pulsations and also found in the JT-60U database. Based on an analysis of these results, we have then shown that these pulses and phase jumps can be explained as the result of the beating between EPMs that coexist in the same radial domain but at different frequencies.

In the simulated scenario, which was dominated by beating EPMs, it was difficult to of the oscillation frequency of a well-defined mode (or wave packet). Thus, we cannot rule out the possibility that, during each pulse (or beat), the oscillation frequency may evolve differently from the long-term trend: it may remain constant or even chirp in the opposite direction temporarily. demonstrates that MEGA is capable of simulating with reasonable accuracy the subtle details of the phase space dynamics of fast ions that interact nonlinearly with the field of shear Alfvén waves. The results reported here are regarded as a successful validation of the code with respect to low-amplitude long-wavelength shear Alfvén mode dynamics on time scales in the range $0.01-10 \mathrm{~ms}$.

Meanwhile, the validation with respect to large-amplitude MHD fluctuations namely, ALEs and their periodic recurrence on the 10-100 ms time scale - has also made good progress [33], as will be reported elsewhere in detail. Since ALEs cause avalanche-like fast ion transport $\left(\Delta \beta_{\mathrm{h} 0} / \beta_{\mathrm{h} 0} \sim 20 \%\right)$ within a short time interval $(<1$ $\mathrm{ms}$ ), a thorough understanding is desirable.

Numerical experiments in the form of first-principle simulations such as those reported here produce results whose analysis may help to clarify various open physics questions, improve our physical picture of fast ion dynamics and, thereby, bridge the gap between theory and experiments. Results of an orbit-based resonance analysis $[20,32,38]$ applied to the chirping and beating EPM dynamics in the present selfconsistent long-time simulations will be reported elsewhere. The trigger mechanism responsible for the onset of ALEs is also under investigation. We anticipate that the 
cumulative changes in the fast ion distribution caused by the chirping $n=1 \mathrm{EPMs}$ studied here play a key role in setting up the system so that it becomes susceptible to ALEs.

\section{Acknowledgments}

One of the authors (A.B.) would like to thank K. Tani for helpful explanations concerning technical details of OFMC simulations. A.B. also thanks B.D. Scott for a helpful comment concerning $\boldsymbol{v}_{E}^{*}$ in Eq. (13). This work has been partially supported by Grants-in-Aid for Scientific Research from the Japan Society for the Promotion of Science (JSPS), Grant No. 25820443 and 16K18341, and NIFS Collaborative Research Program (NIFS12KNTT016). The simulations were carried out using the supercomputer system HELIOS at IFERC, Aomori, Japan, under the Broader Approach collaboration between Euratom and Japan, implemented by Fusion for Energy and QST.

\section{Appendix A. Parameter sensitivity issues and the role of self-organization}

JT-60U shot E039672 was one of the few discharges, where the N-NB system successfully performance fast ion source in our simulation, one may question the comparability of the simulation results with observations made in most other shots, where N-NBs could be operated reliably only at reduced energy and power, around $E_{0} \approx 300-360 \mathrm{keV}$ and 2-4 MW. and current-driven macro- and micro-instabilities. Such processes may give the plasma profiles a significant degree of resilience that allows them to persists under a wide range 
of driving conditions. The existence of such profile resilience in various contexts is currently a hot topic in fusion plasma research, including fast ion physics [39].

Concerning the beam energy, it is indeed likely that the stability of certain modes depends strongly on whether fast ions in the 300-400 keV energy range are present or

5 not. However, if we consider the possibility that the main players on the MHD side are not discrete Alfvén eigenmodes but EPMs — which was the case in our simulations the wide range of possible resonances offered by the continuous spectra of shear Alfvén waves may reduce the sensitivity of the phenomena with respect to the beam energy.

Under this premise, we believe that our comparisons between result of JT-60U experiments and self-consistent MEGA simulations constitute a well-posed validation study, in spite of all the difficulties one faces in the setup of the simulation scenarios realistically. In other words, the likely differences between the simulated and experimental scenarios do not mean that we should expect MEGA result to differ from JT-60U observations. Instead, the robustness of the experimental observations means that MEGA should reproduce many qualitative and quantitative features of the JT-60U results. The fact that MEGA does indeed reproduce experimental results in the present work and related studies [33] allows us to conclude that MEGA accurately captures essential physical processes at the relevant levels of details.

\section{Appendix B. Estimation of the safety factor using MHD spectroscopy}

20 The shape of the $q$ profile shown in Fig. 3(b) was largely determined on the basis of the following observations.

During the initial current ramp-up phase, which lasts for about $4 \mathrm{~s}$, the magnetic probe signal shows rapidly chirping modes, whose mean frequencies sweep slowly up and down (e.g., see Fig. 2 in Ref. [3] for shot E032359). It is thought that the slow sweeps reflect primarily the evolution of the $q$ profile as follows. The upward sweep is assumed to follow the accumulation point of the $(m, n)=(2,1)$ harmonic of the shear Alfvén continuum at the off-axis minimum of $q$, so its presence suggests that $q_{\text {min }}$ has dropped below 2. The interferometer signal (e.g., see Fig. 6 in Ref. [2] for shot E032359) revealed another upward sweep, presumably with $(m, n)=(3,1)$. The frequency difference between the two up-sweeping signals gives us an idea of how far apart the $q=2$ and $q=3$ surfaces are.

Around $t \approx 4 \mathrm{~s}$, the downward sweeping signal (presumably core-localized) and the upward sweeping signal (assumed to follow $q_{\text {min }}$ off-axis) end up at a similar frequency around 50-60 kHz. The associated rapid upward chirps disappear soon after that in most shots. Hence, it can be assumed that $q_{\text {min }}$ has propagated to the plasma center, so that we have $q_{0} \approx q_{\text {min }}<2$ with little or no shear reversal. After that, the period $4-5 \mathrm{~s}$ is typically governed by rapidly chirping modes with relatively constant mean frequency that occur in bursts at 5-10 ms intervals, and abrupt large events (ALE) that occur at 40-60 ms intervals (see Figs. 1 and 2).

40 Later, around $t \approx 5 \mathrm{~s}$, internal kink ("sawtooth") activity becomes visible, which 
indicates that the safety factor in the core has decreased to $q \lesssim 1$.

On the basis of these observations and some additional constraints (e.g., the total plasma current $I_{\mathrm{p}}$ ), the $q$ profile shown in Fig. 3(b) was constructed. It is assumed to be typical for many discharges during the phase where chirping modes and ALEs are strong up-chirping signals, which indicate the survival of a central region with negative magnetic shear.

\section{Appendix C. Estimation of the beta profile}

The overall magnitude of the beta value is estimated from the diamagnetic a thermodynamic equilibrium. The shape of the pressure profile shown in Fig. 3(b) in the form of the toroidal beta $\beta(\rho)=2 \mu_{0} P(\rho) / B_{0}^{2}$ with $P=P_{\mathrm{b}}+P_{\mathrm{h}}$, was determined as follows.

The thermal bulk plasma pressure $P_{\mathrm{b}}$ could be estimated from profiles of the and $T_{\mathrm{i}}$ could be determined relatively accurately using direct measurements combined with semi-empirical transport models. The ion density profile $n_{\mathrm{i}}$ is less accurate since it has to be estimated on the basis of the impurity content (mostly carbon), which is known only vaguely. Overall, it is thought that $P_{\mathrm{b}}$ is accurate to within $20 \%$. simplification is expected to fall within the $20 \%$ tolerance associated with the uncertain shape of the $P_{\mathrm{h}}$ profile. 


\section{References}

[1] Y. Kusama, G.J. Kramer, H. Kimura, M. Saigusa, T. Ozeki, K. Tobita, T. Oikawa, K. Shinohara, T. Kondoh, and M. Moriyama et al. Characteristics of Alfvén eigenmodes, burst modes and chirping modes in the Alfvén frequency range driven by negative ion based neutral beam injection in JT-60U. Nucl. Fusion, 39(11Y):1837, 1999.

[2] K. Shinohara, Y. Kusama, M. Takechi, A. Morioka, M. Ishikawa, N. Oyama, K. Tobita, T. Ozeki, S. Takeji, and S. Moriyama et al. Alfvén eigenmodes driven by Alfvénic beam ions in JT-60U. Nucl. Fusion, 41(5):603, 2001.

[3] K. Shinohara, M. Takechi, M. Ishikawa, Y. Kusama, A. Morioka, N. Oyama, K. Tobita, T. Ozeki, the JT-60 Team, N.N. Gorelenkov, C.Z. Cheng, G.J. Kramer, and R. Nazikian. Recent progress of Alfvén eigenmode experiments using N-NB in JT-60U tokamak. Nucl. Fusion, 42:942, 2002.

[4] K. Shinohara, M. Takechi, M. Ishikawa, Y. Kusama, K. Tsuzuki, K. Urata, H. Kawashima, K. Tobita, A. Fukuyama, and C.Z. Cheng et al. Energetic particle physics in JT-60U and JFT-2M. Plasma Phys. Control. Fusion, 46(7):S31, 2004.

[5] M. Ishikawa, M. Takechi, K. Shinohara, Y. Kusama, C.Z. Cheng, G. Matsunaga, Y. Todo, N.N. Gorelenkov, G.J. Kramer, and R. Nazikian et al. Energetic ion transport by abrupt large-amplitude events induced by negative-ion-based neutral beam injection in the JT-60U. Nucl. Fusion, 45(12):1474, 2005.

[6] W. Park, S. Parker, H. Biglari, M. Chance, L. Chen, C.Z. Cheng, T.S. Hahm, W.W. Lee, R. Kulsrud, D. Monticello, L. Sugiyama, and R. White. Three-dimensional hybrid gyrokinetic-magnetohydrodynamics simulation. Phys. Fluids B, 4:2033, 1992.

[7] J. Lang, G.-Y. Fu, and Y. Chen. Nonlinear simulation of toroidal Alfvén eigenmode with source and sink. Phys. Plasmas, 17(4):042309, 2010.

[8] Y. Todo, H.L. Berk, and B.N. Breizman. Simulation of alfvén eigenmode bursts using a hybrid code for nonlinear magnetohydrodynamics and energetic particles. Nucl. Fusion, 52:033003, 2012.

[9] Y. Todo and T. Sato. Linear and nonlinear particle-magnetohydrodynamic simulations of the toroidal Alfvén eigenmode. Phys. Plasmas, 5(5):1321, 1998.

[10] Y. Todo, K. Shinohara, M. Takechi, and M. Ishikawa. Nonlocal energetic particle mode in a JT-60U plasma. Phys. Plasmas, 12(1):012503, 2005.

[11] Y. Todo, M.A. Van Zeeland, A. Bierwage, and W.W. Heidbrink. Multi-phase simulation of fast ion profile flattening due to Alfvén eigenmodes in a DIII-D experiment. Nucl. Fusion, 54:104012, 2014.

[12] Y. Todo, M.A. Van Zeeland, A. Bierwage, W.W. Heidbrink, and M.E. Austin. Validation of comprehensive magnetohydrodynamic hybrid simulations for Alfvén eigenmode induced energetic particle transport in DIII-D plasmas. Nucl. Fusion, 55(7):073020, 2015.

[13] S. Briguglio, G. Fogaccia, G. Vlad, F. Zonca, K. Shinohara, M. Ishikawa, and 
M. Takechi. Particle simulation of bursting Alfvén modes in jt-60u. Phys. Plasmas, 14(5):055904, 2007.

[14] R.F. Heeter, A.F. Fasoli, and S.E. Sharapov. Chaotic regime of Alfvén eigenmode wave-particle interaction. Phys. Rev. Lett., 85(15):3177, 2000.

5 [15] W. Chen, L.M. Yu, Y. Liu, X.T. Ding, H.S. Xie, J. Zhu, L.M. Yu, X.Q., J.X. Li, Y.G. Li, D.L. Yu, Z.B. Shi, X.M. Song, J.Y. Cao, S.D. Song, Y.B. Dong, W.L. Zhong, M. Jiang, Z.Y. Cui, Y. Huang, Y. Zhou, J.Q. Dong, M. Xu, F. Xia, L.W. Yan, Q.W. Yang, X.R. Duan, and the HL-2A Team. Destabilization of reversed shear Alfvén eigenmodes driven by energetic ions during NBI in HL-2A plasmas with $q_{\text {min }} \sim 1$. Nucl. Fusion, 54(10):104002, 2014.

[16] M. Azumi, G. Kurita, T. Matsuura, T. Takeda, Y. Tanaka, and T. Tsunematsu. In Proceedings of the 4th International Symposium on Computational Methods in Applied Science and Engineering, page 335, Paris, 1980.

[17] A. Bierwage, Y. Todo, N. Aiba, and K. Shinohara. Sensitivity study for N-NBdriven modes in JT-60U: Boundary, diffusion, gyroaverage, compressibility. Nucl. Fusion, 56(10):106009, 2016.

[18] A. Bierwage, Y. Todo, N. Aiba, and K. Shinohara. Dynamics of low- $n$ shear Alfvén modes driven by energetic N-NB ions in JT-60U. Nucl. Fusion, 54(10):104001, 2014.

[19] R.G. Littlejohn. Variational principles of guiding centre motion. J. Plasma Phys., 29(1):111, 1983.

[20] A. Bierwage and K. Shinohara. Orbit-based analysis of resonant excitations of Alfvén waves in tokamaks. Phys. Plasmas, 21(11):112116, 2014.

[21] S. Suzuki, T. Shirai, M. Nemoto, K. Tobita, H. Kubo, T. Sugie, A. Sakasai, and Y. Kusama. Attenuation of high-energy neutral hydrogen beams in high-density plasmas. Plasma Phys. Control. Fusion, 40:2097, 1998.

[22] K. Tani, M. Azumi, H. Kishimoto, and S. Tamura. Effect of toroidal field ripple on fast ion behavior in a tokamak. J. Phys. Soc. Japan, 50(5):1726, 1981.

[23] K. Tani and M. Azumi. Simulation studies on alpha-particle-driven current in tokamak reactors. Nucl. Fusion, 48(8):085001, 2008.

[24] A. Bierwage, C. Di Troia, S. Briguglio, and G. Vlad. Orbit-based representation of equilibrium distribution functions for low-noise initialization of kinetic simulations of toroidal plasmas. Comp. Phys. Comm., 183:1107, 2012.

[25] A.H. Boozer and G. Kuo-Petravic. Monte Carlo evaluation of transport coefficients. Phys. Fluids, 24(5):851, 1981.

[26] B.A. Trubnikov. Particle interactions in a fully ionized plasma. Rev. Plasma Phys., 1:105, 1965. [Original text: B.A. Trubnikov, M.A. Leontovich (Ed.), Voprosy Teorii Plasmy, Vypusk 1, Gosatomizdat, Moscow, 1963, pp.98-182].

[27] T.H. Stix. Heating of toroidal plasmas by neutral injection. Plasma Phys., 14:367, 1972.

[28] A. Bierwage. Merging F3D-OFMC and MEGA: Recent progress with hybrid 
simulations including sources, collisions and sinks. 11th Meeting of the ITPA Energetic Particle Topical Group, Beijing, China, September 2013.

[29] A. Bierwage, N. Aiba, Y. Todo, W. Deng, M. Ishikawa, G. Matsunaga, K. Shinohara, and M. Yagi. Multi-time-scale energetic particle dynamics in JT60U simulated with MHD activity, sources and collisions, invited talk 21aC-2-1. In Proceedings of the 31st Annual Meeting of the Japan Society of Plasma Science and and Nuclear Fusion Research, 2014 Plasma Conference, Nov 11-18, 2014, Niigata, Japan, http://www.jspf.or.jp/PLASMA2014/jpn/yokou.html, 2014.

[30] L. Chen. Theory of magnetohydrodynamic instabilities excited by energetic particles in tokamaks. Phys. Plasmas, 1(5):1519, 1994.

[31] Y. Todo, K. Shinohara, M. Takechi, and M. Ishikawa. Computer simulation of frequency sweeping of energetic particle mode in a JT-60U experiment. J. Plasma Fusion Res., 79(11):1107, 2003.

[32] A. Bierwage and K. Shinohara. Orbit-based analysis of nonlinear energetic ion dynamics in tokamaks. II. Mechanisms for rapid chirping and convective amplification. Phys. Plasmas, 23(4):042512, 2016.

[33] A. Bierwage, K. Shinohara, Y. Todo, N. Aiba, G. Matsunaga, M. Takechi, M. Toma, and M. Yagi. First-principle simulations reproduce multiple cycles of abrupt large amplitude events in beam-driven JT-60 plasmas, oral THW/4-3. In Proceedings of the 26th Fusion Energy Conference, Oct 17-22, 2016, Kyoto, Japan, edited by International Atomic Energy Agency (IAEA), Vienna, 2016. To appear.

[34] L. Chen and A. Hasegawa. Kinetic theory of geomagnetic pulsations: 1. Internal excitations by energetic particles. J. Geophys. Res., 96(A2):1503, 1991.

[35] A. Bierwage, Y. Todo, N. Aiba, K. Shinohara, M. Ishikawa, and M. Yagi. Nonlinear hybrid simulations of energetic particle modes in realistic tokamak flux surface geometry. Plasma Fus. Res., 6:2403109, 2011.

[36] A. Bierwage, N. Aiba, Y. Todo, W. Deng, M. Ishikawa, G. Matsunaga, K. Shinohara, and M. Yagi. Nonlinear simulation of energetic particle modes in high-beta tokamak plasma. Plasma Fus. Res., 7:2403081, 2012.

[37] A. Bierwage, K. Shinohara, N. Aiba, and Y. Todo. Role of convective amplification of $n=1$ energetic particle modes for N-NB ion dynamics in JT-60U. Nucl. Fusion, 53(7):073007, 2013.

[38] A. Bierwage and K. Shinohara. Orbit-based analysis of nonlinear energetic ion dynamics in tokamaks. I. Effective mode number profile and resonant frequency tracking. Phys. Plasmas, 23(4):042511, 2016.

[39] Y. Todo, M.A. Van Zeeland, and W.W. Heidbrink. Fast ion profile stiffness due to the resonance overlap of multiple Alfvén eigenmodes. Nucl. Fusion, 56(11):112008, 2016. 